\title{
Non-linear uplift Ansätze for the internal metric and the four-form field-strength of maximal supergravity
}

\author{
Olaf Krüger \\ Max-Planck-Institut für Gravitationsphysik, Albert-Einstein-Institut, \\ Am Mühlenberg 1, D-14476 Potsdam, Germany \\ E-mail: olaf .krueger@aei.mpg.de
}

ABSTRACT: The uplift of $\mathrm{SO}(8)$ gauged $N=8$ supergravity to 11-dimensional supergravity is well studied in the literature. It is given by consistent relations between the respective vector and scalar fields of both theories. For example, recent work provided non-linear uplift Ansätze for the scalar degrees of freedom on the internal manifold: the inverse metric and the three-form flux with mixed index structure. However, one always found the metric of the compactified manifold by inverting the inverse metric - a task that was only possible in particular cases, e.g. for the $\mathrm{G}_{2}, \mathrm{SO}(3) \times \mathrm{SO}(3)$ or $\mathrm{SU}(3) \times \mathrm{U}(1) \times \mathrm{U}(1)$ invariant solutions of 11-dimensional supergravity.

In this paper, I present a direct non-linear uplift Ansatz for the internal metric in terms of the four-dimensional scalars and the Killing forms on the compactified background manifold. Based on this formula, I also find new uplift Ansätze for the warp factor and the full internal three-form flux, as well as for the internal four-form field-strength. The new formula for the four-form only depends on the metric, the flux as well as the fourdimensional scalars and background Killing forms - it does not require to calculate the derivative of the flux. All the Ansätze presented in this work pass a very non-trivial test for a $G_{2}$ invariant solution of 11-dimensional supergravity.

My results may be generalized to other compactifications, e.g. the reduction from type IIB supergravity to five dimensions.

KEYWORDS: Supergravity Models, Global Symmetries

ArXiv EPrint: 1602.03327 


\section{Contents}

1 Introduction 1

2 The uplift of $N=8$ supergravity to 11-dimensional supergravity 4

3 Known Ansätze for $\Delta^{-1} g^{m n}, A_{m n}^{p}$ and $A_{m_{1} \cdots m_{6}}$

4 New non-linear Ansätze for the metric $g_{m n}$, the warp factor $\Delta$ and the $\begin{array}{ll}\text { full internal three-form potential } A_{m n p} & 11\end{array}$

5 A new non-linear Ansatz for the four-form field-strength 14

6 Testing the new uplift Ansätze 16

$\begin{array}{llr}7 & \text { Conclusion } & 20\end{array}$

A Gamma matrices, Killing spinors, Killing vectors and Killing forms of the $S^{7}$

\section{Introduction}

A supergravity theory in $D>4$ dimensions may be related to a four-dimensional theory of gravity coupled to matter. This is the idea of Kaluza-Klein theory: a D-dimensional manifold splits into a four-dimensional and a compact $(D-4)$-dimensional manifold,

$$
\mathcal{M}_{D}=\mathcal{M}_{4} \times \mathcal{M}_{D-4} .
$$

This splitting is called compactification of the $(D-4)$ extra dimensions. An action including the $D$-dimensional Einstein-Hilbert term is given by

$$
S=\int\left(R_{D}+\ldots\right) \mathrm{d} V,
$$

where $R_{D}$ denotes the Ricci scalar in $D$ dimensions. For a consistent compactification, eq. (1.2) contains the four-dimensional Einstein-Hilbert action. All other terms correspond to matter. For example, T. Kaluza and O. Klein presented one of the first attempts to unify gravity and electromagnetism $[1,2]$. They constructed a five-dimensional theory of gravity,

$$
S_{5}=\int R_{5} \mathrm{~d} V
$$

such that the extra components of the metric were given by a photon and a scalar field. In that case, the fifth dimension was compactified on a circle,

$$
\mathcal{M}_{5}=\mathcal{M}_{4} \times S^{1} .
$$


A physicist naturally is in another situation. He 'observes' a four-dimensional theory of gravity coupled to matter and may ask the following question: is there a higher-dimensional theory, which consistently reduces to the observed theory via compactification of the extra dimensions? This is called an uplift: one constructs the $D$-dimensional fields (e.g. the metric) out of a given four-dimensional theory of gravity. The main task in establishing such a program is to find Ansätze for the $D$-dimensional fields in terms of the four-dimensional ones, such that they satisfy the higher-dimensional equations of motion. The uplift is consistent only when the latter is satisfied.

One of the few known examples is the uplift of $N=8$ supergravity to 11-dimensional supergravity. $N=8$ supergravity represents the low-energy limit of string theory. It is the maximally supersymmetric theory of gravity and contains a local SU(8) gauge symmetry. It was first investigated in the beginning of the 80s [3,4]. At the same time, 11-dimensional supergravity was developed [5], which is the highest dimensional supergravity theory [6]. The respective Lagrangian is also locally $\mathrm{SU}(8)$ gauge invariant.

11-dimensional supergravity may spontaneously compactify to $\mathrm{SO}(8)$ gauged $N=8$ supergravity [7-10]. The seven extra dimensions therefore compactify on a seven-sphere, ${ }^{1}$

$$
\mathcal{M}_{11}=\mathcal{M}_{4} \times S^{7}
$$

This work is based on the uplift of $\mathrm{SO}(8)$ gauged $N=8$ supergravity to 11-dimensional supergravity [9, 11-14]. It is given by non-linear Ansätze for the 11-dimensional scalar and vector fields in terms of the four-dimensional ones. These include the correct relations between the 28 vector fields of 11-dimensional supergravity and the 28 vectors of $N=8$ supergravity. On the other hand, the 70 scalar degrees of freedom of 11-dimensional supergravity are contained in certain fields that are defined on the internal space (a deformed seven-sphere): the metric $g_{m n}$, the three-form potential $A_{m n p}$ and the six-form potential $A_{m_{1} \cdots m_{6}}$. For the complete uplift, these fields must be related to the 35 scalars $u_{i j}{ }^{I J}$ and pseudo-scalars $v_{i j} I J$ of $N=8$ supergravity.

There is an old explicit formula for the inverse metric $\Delta^{-1} g^{m n}[15]$, as well as nonlinear Ansätze for the full internal six-form potential and the three-form flux with mixed index-structure [14]. There are two technical problems arising here: first, one must invert $\Delta^{-1} g^{m n}$ 'by hand' in order to obtain $\Delta g_{m n}$. Secondly, one must extract the warp factor $\Delta$ from these expressions by computing their determinants. Both, the inversion of the metric and the calculation of the warp factor can only be done in particular cases, e.g. when the theory is $\mathrm{G}_{2}, \mathrm{SO}(3) \times \mathrm{SO}(3)$ or $\mathrm{SU}(3) \times \mathrm{U}(1) \times \mathrm{U}(1)$ invariant [16-19]. Only in such cases, it is then possible to compute the full internal three-form potential $A_{m n p}$.

In this paper, I present a new simple non-linear Ansatz for the full internal metric $g_{m n}$, i.e.

$$
\Delta^{-2} g_{m n}(x, y)=\frac{1}{12}\left(\mathcal{A}_{m i j k l}-\mathcal{B}_{m i j k l}\right)\left(\mathcal{A}_{n}{ }^{i j k l}-\mathcal{B}_{n}{ }^{i j k l}\right)(x, y) .
$$

The tensors $\mathcal{A}_{m}{ }^{i j k l}$ and $\mathcal{B}_{m}{ }^{i j k l}$ are given in terms of the Killing forms on the seven-sphere and the four-dimensional scalar fields (eqs. (4.4)-(4.7)). In combination with the previous

\footnotetext{
${ }^{1} \mathrm{SO}(8)$ is the isometry group for $S^{7}$.
} 
uplift formulas for the inverse metric and the three-form with mixed index structure, $I$ also find new non-linear Ansätze for the warp factor and the full internal three-form potential $A_{m n p}$. They are given by

$$
\begin{aligned}
\Delta^{-3}(x, y) & =\frac{1}{28 \cdot 4 !} \mathcal{C}_{i j}{ }^{k l m n}(x, y) \mathcal{C}^{i j}{ }_{k l m n}(x, y), \\
\Delta^{-3} A_{m n p}(x, y) & =-\frac{\sqrt{2} i}{48 \cdot 4 !} K_{m n}{ }^{I J}(y)\left(u^{i j}{ }_{I J}-v^{i j I J}\right)(x) \mathcal{C}_{i j}{ }^{q r s t}(x, y)\left(\mathcal{A}_{p q r s t}-\mathcal{B}_{p q r s t}\right)(x, y),
\end{aligned}
$$

where the tensor $\mathcal{C}_{p q}{ }^{i j k l}$ is defined similarly to $\mathcal{A}_{m}{ }^{i j k l}$ and $\mathcal{B}_{m}{ }^{i j k l}$ in eq. (4.10). The twoforms $K_{m n}{ }^{I J}$ denote the derivative of the Killing vectors $K_{m}{ }^{I J}$ on the round seven-sphere.

During completion of this paper, a work by Oscar Varela derived similar coordinatefree Ansätze for the metric, the warp factor and the flux [20]. These expressions however, are given in a different form that is based on the tensor hierarchy formalism of gauged supergravity (see eqs. (24-26) of [20]). This makes it complicated to actually compare my formulas to those of Varela's work. In order to illustrate the simplicity of the Ansätze above, I test them for a $\mathrm{G}_{2}$ invariant solution of 11-dimensional supergravity. This essential part of the present work is done in section 6. It turns out that the new formulas in eqs. (1.6)-(1.8) appear to be very suitable for this test.

In the second part of this paper, I derive a new uplift Ansatz for the internal four-form field-strength

$$
F_{m n p q}=4 ! \stackrel{\circ}{D}_{[m} A_{n p q]} .
$$

Here, $\stackrel{\circ}{D}_{m}$ denotes the covariant derivative with respect to the internal background metric $\stackrel{\circ}{m}_{m n}$. So far, eq. (1.9) could only be used in particular cases — when an explicit expression for the internal three-form potential was already given. However, it was rather complicated to compute the derivative of $A_{m n p}$ in such cases, for example to find the $\mathrm{G}_{2}$ or $\mathrm{SO}(3) \times \mathrm{SO}(3)$ invariant solutions of 11-dimensional supergravity [16, 17]. With the new general Ansatz for $A_{m n p}$ above, I derive a simple direct formula for the four-form field-strength, i.e.

$$
\begin{aligned}
F_{m n p q}= & m_{7} \Delta \stackrel{\circ}{g}_{s[m}\left(4 \epsilon_{n p q] r_{1} r_{2} r_{3}} A^{r_{1} r_{2} r_{3}}\right. \\
& \left.-3 g_{n|t|} A_{p q] r} K^{r s I J} K^{t K L}\left(u_{i j}^{I J}+v_{i j I J}\right)\left(u^{i j} K L+v^{i j K L}\right)\right) .
\end{aligned}
$$

Here, $m_{7}$ denotes the inverse $S^{7}$ radius and $\epsilon_{r_{1} \cdots r_{7}}$ is the internal $\epsilon$-tensor.

A formula for the complete four-form field-strength occurs in eq. (28) of Varela's work [20]. Again, it is hard to compare both formulas because the expression in [20] is given in a form based on the tensor hierarchy formalism of gauged supergravity. In section 6, I will demonstrate once more that the present Ansatz above is given in a very convenient form - it can be directly used for a test against the $G_{2}$ invariant solution of 11-dimensional supergravity. 
The new non-linear Ansatz in eq. (1.10) provides another remarkable result: the above expression is 'almost' covariant ${ }^{2}$, which means that raising the indices is simple,

$$
\begin{aligned}
F^{m n p q}= & m_{7} \Delta \stackrel{\circ}{s t}_{s t} g^{t[m}\left(4 \epsilon^{n p q] r_{1} r_{2} r_{3} s} A_{r_{1} r_{2} r_{3}}\right. \\
& \left.-3 A^{n p}{ }_{r} K^{q] I J} K^{r s K L}\left(u^{i j}{ }_{I J}+v^{i j I J}\right)\left(u_{i j}{ }^{K L}+v_{i j K L}\right)\right) .
\end{aligned}
$$

Up to now, it was far more complicated to derive $F^{m n p q}$ - by raising each single index of $F_{m n p q}$ with the explicit expression for the inverse metric $g^{m n}$. For example, this was one of the hardest tasks in verifying the $\mathrm{SO}(3) \times \mathrm{SO}(3)$ invariant solution of 11-dimensional supergravity [17]. In the case of maximally symmetric spacetimes, these results can be used to compute the components of the Ricci tensor via the equations of motion.

In the next section, I collect the main steps to find the consistent uplift of $N=8$ supergravity to 11-dimensional supergravity. In section 3, I re-derive the known nonlinear Ansätze for the inverse metric $\Delta^{-1} g^{m n}$, the three-form with mixed index structure $A_{m n}{ }^{p}$ and the six-form potential $A_{m_{1} \cdots m_{6}}$. In section 4, I present the new uplift Ansätze for the metric $g_{m n}$, the warp factor $\Delta$ and the full internal three-form potential $A_{m n p}$. Furthermore, I find the new non-linear Ansatz for the four-form field-strength $\left(F_{m n p q}\right.$ and $F^{m n p q}$ ) in section 5. In section 6 , I test the new uplift Ansätze for the $\mathrm{G}_{2}$ invariant solution of 11-dimensional supergravity: I compute the metric and the four-form fieldstrength using the new formulas in eqs. (1.6), $(1.10)^{3}$ and compare with the results of [16]. Finally, I conclude in section 7.

\section{The uplift of $N=8$ supergravity to 11-dimensional supergravity}

The bosonic field content of 11-dimensional supergravity is an elfbein $E_{M}{ }^{A}(x, y)$ and a three-form potential $A_{M N P}(x, y)$. The set of coordinates splits into four spacetime (external) coordinates $x$ and seven internal coordinates $y$. Capital Roman letters denote 11dimensional indices. These split into external (Greek letters) and internal indices (lower case Roman letters). As a rule of thumb: letters from the middle of an alphabet always denote curved spacetime indices and letters from the beginning of an alphabet are the corresponding tangent space indices.

The bosonic Lagrangian of 11-dimensional supergravity is written in terms of the elfbein, the three-form potential and the four-form field-strength [7]. The latter is defined by

$$
F_{(4)}=\mathrm{d} A_{(3)} \quad \Leftrightarrow \quad F_{M N P Q}=4 ! \partial_{[M} A_{N P Q]} .
$$

The Lagrangian can also be written in terms of dual fields [21]: for example, one could replace $F_{(4)}$ by its dual seven-form

$$
F_{(7)}=\star F_{(4)}
$$

\footnotetext{
${ }^{2}$ Indices of $\stackrel{\circ}{g}_{m n}$ and the Killing forms are raised and lowered with the background metric. All other tensors are covariant.

${ }^{3} \mathrm{~A}$ combination of the old Ansätze for $\Delta^{-1} g^{m n}, A_{m n}{ }^{p}$ and the new metric Ansatz yields the new formulas for the warp factor $\Delta$ and the internal three-form $A_{m n p}$. The old expressions for the inverse metric and the three-form potential with mixed index structure have already been tested in [16]. Hence, it suffices to test the Ansätze for the metric $g_{m n}$ and the four-form field-strength $F_{m n p q}$ for a $\mathrm{G}_{2}$ invariant solution of 11-dimensional supergravity.
} 
and the three-form potential by its dual six-form $A_{M_{1} \cdots M_{6}}$. The latter is the potential for the dual seven-form field-strength,

$$
F_{(7)}=\mathrm{d} A_{(6)}+3 \sqrt{2} A_{(3)} \wedge F_{(4)}+\text { fermionic terms. }
$$

Later, one needs the six-form potential to describe certain vector and scalar degrees of freedom.

Let us count the scalar and vector fields in 11-dimensional supergravity. The elfbein is given by

$$
E_{M}{ }^{A}=\left(\begin{array}{cc}
e_{\mu}{ }^{\alpha} & B_{\mu}{ }^{m} e_{m}{ }^{a} \\
0 & e_{m}{ }^{a}
\end{array}\right) .
$$

It contains the vierbein $e_{\mu}{ }^{\alpha}(x, y)$, seven vectors $B_{\mu}{ }^{m}(x, y)$ and 28 scalar fields $e_{m}{ }^{a}(x, y)$. On the other hand, the three-form potential splits into the components

$$
A_{M N P}=\left(A_{\mu \nu \rho}, A_{\mu \nu m}, A_{\mu m n}, A_{m n p}\right) .
$$

There are 21 vector fields in $A_{\mu m n}(x, y)$. Furthermore, $A_{\mu \nu m}(x, y)$ contains seven and $A_{m n p}(x, y) 35$ scalar degrees of freedom. The remaining components $A_{\mu \nu \rho}(x, y)$ represent the potential for the external field-strength

$$
F_{\mu \nu \rho \sigma}(x, y)=4 ! \partial_{[\mu} A_{\nu \rho \sigma]}(x, y)
$$

and hence, contain no more scalar or vector degrees of freedom. This is because for all dimensional reductions,

$$
F_{\mu \nu \rho \sigma}(x, y)=i \mathfrak{f}_{\mathrm{FR}}(x, y) \stackrel{\circ}{\mu \nu \rho \sigma}
$$

The Freund-Rubin parameter $\mathfrak{f}_{\mathrm{FR}}$ is constant for Freund-Rubin compactifications [22] and $\stackrel{\circ}{\eta}_{\mu \nu \rho \sigma}$ represents the volume form in four dimensions. All in all, there are $7+21=28$ vectors and $28+7+35=70$ scalar degrees of freedom in 11-dimensional supergravity.

The bosonic field content of $N=8$ supergravity is a vierbein $\stackrel{\circ}{\mu}_{\mu}{ }^{\alpha}(x), 28$ 'electric' vector fields $A_{\mu}{ }^{I J}(x)$ as well as 35 scalar and 35 pseudo-scalar fields $u_{i j}{ }^{I J}(x), v_{i j}{ }_{I J}(x)$. All these fields only depend on the four spacetime coordinates $x$. The (antisymmetric) bi-vector indices $I J$ belong to the 28-dimensional representation of $\mathrm{SL}(8, \mathbb{R})$ and the (antisymmetric) bi-vector indices $i j$ belong to the 28 -dimensional representation of the local $\mathrm{SU}(8)$. The bosonic degrees of freedom of both, $N=8$ supergravity and 11-dimensional supergravity coincide. This is at least, necessary for a consistent uplift.

In order to uplift $N=8$ supergravity to 11-dimensional supergravity, one must explicitly relate the vierbeine, as well as the scalar and vector fields of both theories to each other. In the following, I will restrict to the $S^{7}$ compactification [10]. The matching was found by comparing the supersymmetry transformations of the four- and 11-dimensional fields [14, 23]. It is based on a global $\mathrm{E}_{7(7)}$ symmetry in $N=8$ supergravity [3]. $\mathrm{E}_{7(7)}$ is not a symmetry of 11-dimensional supergravity. However, one may emphasize the respective $\mathrm{E}_{7(7)}$ structures as much as possible in order to compare the fields with those of $N=8$ supergravity. 
The correct relation between the vierbeine of $N=8$ supergravity and 11-dimensional supergravity is

$$
e_{\mu}^{\alpha}(x, y)=\Delta(x, y)^{-1 / 2} \grave{e}_{\mu}^{\alpha}(x) .
$$

The proportionality factor $\Delta(x, y)$ is called the warp factor. Let $\stackrel{e}{m}_{m}{ }^{a}$ be the siebenbein for the round seven-sphere and $\stackrel{\circ}{g}_{m n}$ denote the respective background metric and let $g_{m n}$ be the full internal metric of the deformed $S^{7}[12]$,

$$
\stackrel{\circ}{g}_{m n}=\stackrel{\circ}{e}_{m}^{a} \stackrel{\circ}{e}_{n a}, \quad g_{m n}=e_{m}{ }^{a} e_{n a} .
$$

Then, the warp factor is defined by

$$
\Delta=\frac{\operatorname{det}\left(e_{m}{ }^{a}\right)}{\operatorname{det}\left(\stackrel{\circ}{e}_{m}^{a}\right)}=\sqrt{\frac{\operatorname{det}\left(g_{m n}\right)}{\operatorname{det}\left(\stackrel{\circ}{g}_{m n}\right)}} .
$$

In order to match the scalar degrees of freedom, one first observes that the 35 scalars and 35 pseudo-scalars of $N=8$ supergravity parametrize an element of $\mathrm{E}_{7} / \mathrm{SU}(8)$. This co-set space is indeed, 70-dimensional. Both, scalars and pseudo-scalars together form an element $\hat{\mathcal{V}}^{\mathcal{M}}{ }_{i j}(x)$ in the fundamental representation $\mathbf{5 6}$ of $\mathrm{E}_{7(7)}$. Its $\mathrm{SL}(8, \mathbb{R})$ decomposition is given by

$$
\begin{aligned}
\hat{\mathcal{V}}^{\mathcal{M}}{ }_{i j} & =\left(\frac{i}{\sqrt{2}}\left(u_{i j}{ }^{I J}+v_{i j I J}\right),-\frac{1}{\sqrt{2}}\left(u_{i j}{ }^{I J}-v_{i j I J}\right)\right), \\
\mathbf{5 6} & \rightarrow \mathbf{2 8} \oplus \overline{\mathbf{2 8}} .
\end{aligned}
$$

The 56 representation is labeled by indices $\mathcal{M}, \mathcal{N}, \ldots$, which are raised and lowered with the symplectic form $\Omega_{\mathcal{M N}}$ (see [3]). The SU(8) indices $i j$ are raised and lowered via complex conjugation,

$$
u_{I J}^{i j}=\left(u_{i j}^{I J}\right)^{*}, \quad v^{i j I J}=\left(v_{i j I J}\right)^{*} .
$$

One also writes the scalar fields of 11-dimensional supergravity in an $\mathrm{E}_{7(7)}$ covariant way. Therefore, it is convenient to describe all scalars by the fields $e_{m}{ }^{a}, A_{m_{1} \cdots m_{6}}$ and $A_{m n p}$ (rather than using $A_{\mu \nu m}$ ). Indeed, the internal dual six-form potential $A_{m_{1} \cdots m_{6}}$ contains the same scalar degrees of freedom as $A_{\mu \nu m}$. In a second step, one converts this scalar field content $\left(e_{m}{ }^{a}, A_{m_{1} \cdots m_{6}}\right.$ and $\left.A_{m n p}\right)$ into components of a '56-bein' of $\mathrm{E}_{7(7)}$, i.e. $[13,24]$

$$
\begin{aligned}
\mathcal{V}_{A B}^{m}= & -\frac{\sqrt{2}}{8} \Delta^{-1 / 2} \Gamma_{A B}^{m}, \\
\mathcal{V}_{m n A B}= & -\frac{\sqrt{2}}{8} \Delta^{-1 / 2}\left(\Gamma_{m n} A B+6 \sqrt{2} A_{m n p} \Gamma_{A B}^{p}\right), \\
\mathcal{V}_{A B}^{m n}= & -\frac{\sqrt{2}}{8} \cdot \frac{1}{5 !} \grave{\eta}^{m n p_{1} \cdots p_{5}} \Delta^{-1 / 2}\left[\Gamma_{p_{1} \cdots p_{5} A B}+60 \sqrt{2} A_{p_{1} p_{2} p_{3}} \Gamma_{p_{4} p_{5} A B}\right. \\
& \left.-6 ! \sqrt{2}\left(A_{q p_{1} \cdots p_{5}}-\frac{\sqrt{2}}{4} A_{q p_{1} p_{2}} A_{p_{3} p_{4} p_{5}}\right) \Gamma_{A B}^{q}\right],
\end{aligned}
$$




$$
\begin{aligned}
\mathcal{V}_{m A B}= & -\frac{\sqrt{2}}{8} \cdot \frac{1}{7 !} \grave{\eta}^{p_{1} \cdots p_{7}} \Delta^{-1 / 2}\left[\left(\Gamma_{p_{1} \cdots p_{7}} \Gamma_{m}\right)_{A B}+126 \sqrt{2} A_{m p_{1} p_{2}} \Gamma_{p_{3} \cdots p_{7} A B} A\right. \\
& +3 \sqrt{2} \cdot 7 !\left(A_{m p_{1} \cdots p_{5}}+\frac{\sqrt{2}}{4} A_{m p_{1} p_{2}} A_{p_{3} p_{4} p_{5}}\right) \Gamma_{p_{6} p_{7} A B} \\
& \left.+\frac{9 !}{2}\left(A_{m p_{1} \cdots p_{5}}+\frac{\sqrt{2}}{12} A_{m p_{1} p_{2}} A_{p_{3} p_{4} p_{5}}\right) A_{p_{6} p_{7} q} \Gamma_{A B}^{q}\right] .
\end{aligned}
$$

These components constitute the GL $(7, \mathbb{R})$ decomposition of the 56-bein

$$
\begin{aligned}
\mathcal{V}^{\mathcal{M}_{A B}} & =\left(\mathcal{V}^{m}{ }_{A B}, \mathcal{V}_{m n A B}, \mathcal{V}^{m n}{ }_{A B}, \mathcal{V}_{m A B}\right), \\
\mathbf{5 6} & \rightarrow \mathbf{7} \oplus \mathbf{2 1} \oplus \overline{\mathbf{2 1}} \oplus \overline{\mathbf{7}}
\end{aligned}
$$

The $\mathrm{SU}(8)$ indices $A, B, \ldots$ are raised and lowered by complex conjugation ${ }^{4}$ and the $8 \times 8$ $\Gamma$-matrices are defined in appendix A.

The correct relation between the 56-bein in 11 dimensions and the four-dimensional scalars $\hat{\mathcal{V}}$ of $N=8$ supergravity was found by considering the respective supersymmetry transformations [14]..$^{5}$ It is given by

$$
\mathcal{V}^{\mathcal{M}}{ }_{A B}(x, y)=\mathcal{R}^{\mathcal{M}} \mathcal{N}(y) \eta_{A}^{i}(y) \eta_{B}^{j}(y) \hat{\mathcal{V}}^{\mathcal{N}}{ }_{i j}(x)
$$

Here, $\eta_{A}^{i}$ are the eight Killing spinors defined on the internal geometry. The upper index $\mathcal{M}$ of the transformation matrix $\mathcal{R}^{\mathcal{M}}{ }_{\mathcal{N}}$ is decomposed under $\mathrm{GL}(7, \mathbb{R})$ (eq. (2.19)) whereas the lower index $\mathcal{N}$ is decomposed under $\mathrm{SL}(8, \mathbb{R})$ (eq. (2.12)),

$$
\mathcal{R}^{\mathcal{M}_{\mathcal{N}}}=\left(\begin{array}{cc}
\mathcal{R}^{m}{ }_{I J} & \mathcal{R}^{m I J} \\
\mathcal{R}_{m n I J} & \mathcal{R}_{m n}{ }^{I J} \\
\mathcal{R}^{m n}{ }_{I J} & \mathcal{R}^{m n I J} \\
\mathcal{R}_{m I J} & \mathcal{R}_{m}{ }^{I J}
\end{array}\right)
$$

The non-zero components are [14]

$$
\begin{aligned}
\mathcal{R}^{m}{ }_{I J}(y) & =\frac{1}{4} K^{m I J}(y), \\
\mathcal{R}_{m n}{ }^{I J}(y) & =\frac{1}{4} K_{m n}{ }^{I J}(y), \\
\mathcal{R}^{m n}{ }_{I J}(y) & =\frac{1}{4}\left(2 \dot{\zeta}^{[m} K^{n] I J}-K^{m n I J}\right)(y), \\
\mathcal{R}_{m}{ }^{I J}(y) & =\frac{1}{4}\left(\dot{\zeta}^{n} K_{m n}{ }^{I J}-K_{m}{ }^{I J}\right)(y) .
\end{aligned}
$$

They depend on the Killing vectors $K_{m}{ }^{I J}(y)$ and -forms $K_{m n}{ }^{I J}(y)$ as well as on the dual volume potential $\check{\zeta}^{m}(y)$ of the seven-sphere. The Killing vectors and -forms are defined

\footnotetext{
${ }^{4}$ It should always be clear from the context whether $A, B, \ldots$ are $\mathrm{SU}(8)$ - or 11-dimensional tangent space indices.

${ }^{5}$ Note that initially, eq. (2.20) follows from the respective uplift relation for the vectors in eq. (2.34).
} 
in appendix A. The (seven dimensional) dual of $\check{\zeta}^{m}(y)$ is the six-form potential for the internal background volume form ${\stackrel{\circ}{m_{1} \cdots m_{7}}}$,

$$
\begin{aligned}
& \dot{\zeta}^{n}=6 \grave{\eta}^{n m_{1} \cdots m_{6}}{\stackrel{\circ}{m_{1} \cdots m_{6}}}, \quad{\stackrel{\circ}{m_{1} \cdots m_{6}}}=\frac{1}{6 \cdot 6 !} \stackrel{\circ}{\eta}_{m_{1} \cdots m_{7}}{\stackrel{\zeta}{m_{7}}}^{m_{7}}, \\
& 7 ! \stackrel{\circ}{D}_{\left[m_{1}\right.} \stackrel{\circ}{\zeta}_{\left.m_{2} \cdots m_{7}\right]}=m_{7} \stackrel{\circ}{\eta}_{m_{1} \cdots m_{7}}
\end{aligned}
$$

Note the non-standard normalization of $\dot{\zeta}^{m}$, which is more convenient for my purposes. $m_{7}$ denotes the inverse radius of the round $S^{7}$.

Using eqs. (2.11), (2.22)-(2.25), one finally finds the components of

$$
\mathcal{V}_{i j}^{\mathcal{M}}(x, y)=\mathcal{R}^{\mathcal{M}}{ }_{\mathcal{N}}(y) \hat{\mathcal{V}}^{\mathcal{N}}{ }_{i j}(x),
$$

namely

$$
\begin{aligned}
\mathcal{V}^{m 8}{ }_{i j}(x, y) & =\frac{\sqrt{2} i}{8} K^{m I J}(y)\left(u_{i j}{ }^{I J}+v_{i j I J}\right)(x), \\
\mathcal{V}_{m n i j}(x, y) & =-\frac{\sqrt{2}}{8} K_{m n}{ }^{I J}(y)\left(u_{i j}{ }^{I J}-v_{i j} I J\right)(x), \\
\mathcal{V}^{m n}{ }_{i j}(x, y) & =\frac{\sqrt{2} i}{8}\left(2 \zeta^{[m} K^{n] I J}-K^{m n I J}\right)(y)\left(u_{i j}{ }^{I J}+v_{i j I J}\right)(x), \\
\mathcal{V}_{m 8 i j}(x, y) & =-\frac{\sqrt{2}}{8}\left(\check{\zeta}^{n} K_{m n}^{I J}-K_{m}^{I J}\right)(y)\left(u_{i j}{ }^{I J}-v_{i j I J}\right)(x) .
\end{aligned}
$$

In order to match the vector degrees of freedom, one first dualizes the 28 'electric' vector fields $A_{\mu}{ }^{I J}(x)$ in $N=8$ supergravity to form 28 'magnetic' vector fields $A_{\mu I J}(x)$. Only electric and magnetic vector fields together fit into the $\mathbf{5 6}$ representation of $\mathrm{E}_{7(7)}$ : they represent the $\mathrm{SL}(8, \mathbb{R})$ decomposition of

$$
A_{\mu}^{\mathcal{M}}=\left(A_{\mu}{ }^{I J}, A_{\mu I J}\right)
$$

along the lines of eq. (2.12). One also extends the 28 vector fields $B_{\mu}{ }^{m}$ and $A_{\mu m n}$ in 11dimensional supergravity such that they fit into the $\mathbf{5 6}$ representation of $E_{7(7)}$. There are 21 dual vectors $A_{\mu m_{1} \cdots m_{5}}$ coming from the six-form potential and seven 'dual graviphotons' that have no physical interpretation [13]. Similar to the case of scalar fields, one defines a 56-bein $B_{\mu}^{\mathcal{M}}$ of $\mathrm{E}_{7(7)}$, which decomposes under $\mathrm{GL}(7, \mathbb{R})$ into the various vector degrees of freedom above. Since this work concentrates on the uplift of the scalar fields, I do not give the explicit $\mathrm{GL}(7, \mathbb{R})$ decomposition for $B_{\mu}{ }^{\mathcal{M}}$ here. The interested reader may have a look at $[13,14,24]$.

The consistent relation between the vector fields $A_{\mu}{ }^{\mathcal{M}}(x)$ of $N=8$ supergravity and the 11-dimensional vectors $B_{\mu}{ }^{\mathcal{M}}(x, y)$ is similar to eq. $(2.20)^{6}$,

$$
B_{\mu}{ }^{\mathcal{M}}(x, y)=\mathcal{R}^{\mathcal{M}}{ }_{\mathcal{N}}(y) A_{\mu}^{\mathcal{N}}(x)
$$

It has also been found by a careful analysis of the supersymmetry transformations in four and 11 dimensions.

\footnotetext{
${ }^{6}$ The last seven components of $B_{\mu}{ }^{\mathcal{M}}$ belong to the non-physical dual graviphotons. Eq. (2.34) therefore, does only make sense in the first 49 components.
} 
Here is a simple example for the readers convenience: the first seven components of $B_{\mu}^{\mathcal{M}}$ are proportional to the vectors $B_{\mu}{ }^{m}$. With eqs. (2.34), (2.22) one then finds the old Ansatz for the vector fields in Kaluza-Klein theory [25], i.e.

$$
B_{\mu}^{m}(x, y) \propto K^{m I J}(y) A_{\mu}^{I J}(x) .
$$

The task of uplifting $N=8$ supergravity to 11-dimensional supergravity is now the following: starting from eqs. (2.20), (2.34), one must seek explicit expressions for the 11dimensional vector and scalar fields in terms of the four-dimensional ones,

$$
\begin{aligned}
& \left(B_{\mu}{ }^{m}, A_{\mu m n}, A_{\mu m_{1} \cdots m_{5}} \text {, dual graviphotons }\right) \quad \Leftrightarrow \quad\left(A_{\mu}^{I J}, A_{\mu I J}\right), \\
& \left(g_{m n}, A_{m n p}, A_{m_{1} \cdots m_{6}}\right) \quad \Leftrightarrow \quad\left(u_{i j}{ }^{I J}, v_{i j I J}\right) .
\end{aligned}
$$

In principle, these relations have been found in $[14,15]$. However, instead of a relation for the metric $g_{m n}(x, y)$, the authors only found an expression for the inverse metric $\Delta^{-1} g^{m n}(x, y)$, scaled with the warp factor. Furthermore, the Ansätze for the three-form and six-form potentials require the full metric $g_{m n}$. Until now, the inversion of $\Delta^{-1} g^{m n}$ is only possible in particular cases, e.g. for $\mathrm{G}_{2}, \mathrm{SO}(3) \times \mathrm{SO}(3)$ or $\mathrm{SU}(3) \times \mathrm{U}(1) \times \mathrm{U}(1)$ invariant solutions [16-19]. Also the warp factor can only be computed from an explicit expression for the metric $g_{m n}$ (by taking the determinant).

The reader familiar with the uplift Ansätze presented in [14] may skip the next section, which repeats the derivation of the known scalar uplifts. Section 4 then presents new non-linear Ansätze for the full internal metric $g_{m n}$, the warp factor $\Delta$ and the internal three-form potential $A_{m n p}$. These hold for the uplift of $N=8$ supergravity to 11-dimensional maximally gauged supergravity, even without further restrictions (such as $\mathrm{G}_{2}, \mathrm{SO}(3) \times \mathrm{SO}(3)$ or $\mathrm{SU}(3) \times \mathrm{U}(1) \times \mathrm{U}(1)$ invariance $)$.

\section{Known Ansätze for $\Delta^{-1} g^{m n}, A_{m n}^{p}$ and $A_{m_{1} \cdots m_{6}}$}

For the readers convenience, I repeat the steps to derive the known uplift relations for the inverse metric $\Delta^{-1} g^{m n}$, the three-form with mixed index structure $A_{m n}{ }^{p}$ and the six-form potential $A_{m_{1} \cdots m_{6}}$. This was done in [14] and is the basis to understand the new Ansätze for the metric $g_{m n}$, the warp factor $\Delta$ and the full internal three-form potential $A_{m n p}$ in section 4 .

The main problem of comparing the vielbein components in eqs. (2.14)-(2.17) and eqs. (2.29) $-(2.32)$ is the occurrence of the Killing spinors in eq. (2.20). However, these are orthonormal and would drop out in non-linear SU(8)-invariant combinations of the vielbeine. For example, let us consider the expression

$$
\mathcal{V}^{m}{ }_{A B} \mathcal{V}^{n A B}=\eta_{A}^{i} \eta_{B}^{j} \mathcal{V}^{m}{ }_{i j} \eta_{k}^{A} \eta_{l}^{B} \mathcal{V}^{n k l}=\mathcal{V}^{m}{ }_{i j} \mathcal{V}^{n i j}
$$

Indeed, the Killing spinors $\eta_{A}^{i}(y)$ drop out. One now uses eq. (2.14) on the l.h.s. and eq. (2.29) on the r.h.s., which results in a non-linear uplift Ansatz for the inverse metric, i.e.

$$
\Delta^{-1} g^{m n}(x, y)=\frac{1}{8} K^{m I J}(y) K^{n K L}(y)\left(u_{i j}^{I J}+v_{i j I J}\right)(x)\left(u_{K L}^{i j}+v^{i j K L}\right)(x) .
$$

Here, I used the Clifford algebra of the $\Gamma$-matrices, given in appendix A. 
In a similar way, one relates

$$
\mathcal{V}_{m n}{ }^{A B} \mathcal{V}^{p 8}{ }_{A B}=\mathcal{V}_{m n}{ }^{i j} \mathcal{V}^{p 8}{ }_{i j},
$$

which yields a non-linear uplift Ansatz for the three-form. Indeed, using eqs. (2.14), (2.15) on the 1.h.s. as well as eqs. (2.29), (2.30) on the r.h.s., one finds

$$
\Delta^{-1} A_{m n}^{p}(x, y)=-\frac{\sqrt{2} i}{96} K_{m n}^{I J}(y) K^{p K L}(y)\left(u^{i j}{ }_{I J}-v^{i j I J}\right)\left(u_{i j}{ }^{K L}+v_{i j} K L\right)(x) .
$$

In order to derive an uplift Ansatz for the internal six-form potential $A_{m_{1} \cdots m_{6}}$, I introduce the (seven dimensional) dual one-form

$$
A^{n}=6 \epsilon^{n m_{1} \cdots m_{6}} A_{m_{1} \cdots m_{6}} .
$$

Similar to the dual volume potential on the round seven-sphere, $\stackrel{\circ}{\zeta}^{m}$, I use a non-standard normalization for later convenience. The six-form potential $A_{(6)}$ is a tensor in the internal space and its (seven dimensional) dual $A_{(1)}$ is constructed with the full $\epsilon$-tensor. However, one can convert this $\epsilon$-tensor to the tensor density $\grave{\eta}(= \pm 1,0)$ using the internal sevenbein $e_{m}{ }^{a}$

$$
\epsilon_{m_{1} \cdots m_{7}}=e_{m_{1}}^{a_{1}} \ldots e_{m_{7}}^{a_{7}}{\stackrel{\circ}{a_{1} \cdots a_{7}}}=\Delta \dot{\eta}_{m_{1} \cdots m_{7}} .
$$

Here, I used the definition of the warp factor in eq. (2.10). Eq. (3.5) then reads

$$
A^{n}=\frac{6}{\Delta} \grave{\eta}^{n m_{1} \cdots m_{6}} A_{m_{1} \cdots m_{6}} \quad \Leftrightarrow \quad A_{m_{1} \cdots m_{6}}=\frac{\Delta}{6 \cdot 6 !}{\stackrel{\circ}{\eta \cdots m_{7}}} A^{m_{7}} .
$$

Note that the indices of the six-form potential and its dual are raised and lowered with the full internal metric.

Now, let us consider the relation

$$
\mathcal{V}^{m n}{ }_{A B} \mathcal{V}^{p 8 A B}=\mathcal{V}^{m n}{ }_{i j} \mathcal{V}^{p 8 i j}
$$

and insert the various vielbein components in eqs. (2.14), (2.16) and eqs. (2.29), (2.31). This gives an equation for $A^{n}$, i.e.

$$
\begin{aligned}
\frac{\sqrt{2}}{9}\left(\Delta A^{[m}+3 \sqrt{2} \zeta^{[m}\right) g^{n] p}= & \stackrel{\circ}{ }^{m n q_{1} \cdots q_{5}} A_{q_{1} q_{2}}^{p} A_{q_{3} q_{4} q_{5}} \\
& +\frac{\Delta}{24} K^{m n I J} K^{p K L}\left(u_{i j}{ }^{I J}+v_{i j I J}\right)\left(u^{i j} K L+v^{i j K L}\right) .
\end{aligned}
$$

When contracting this relation with $g_{n p}$, the first term on the r.h.s. drops out because

$$
A_{[m n p} A_{q r s]}=0 .
$$

One finds

$$
\begin{aligned}
\Delta A^{m}(x, y)+3 \sqrt{2} \grave{\zeta}^{m}(y)= & \frac{\Delta(x, y)}{8 \sqrt{2}} g_{n p}(x, y) K^{m n I J}(y) \\
& \times K^{p K L}(y)\left(u_{i j}{ }^{I J}+v_{i j I J}\right)\left(u^{i j} K L+v^{i j K L}\right)(x)
\end{aligned}
$$


and dualizes this expression using eq. (3.7), (2.26),

$$
A_{m_{1} \cdots m_{6}}+3 \sqrt{2} \stackrel{\circ}{\zeta}_{m_{1} \cdots m_{6}}=\frac{\sqrt{2}}{96 \cdot 6 !} \epsilon_{n m_{1} \cdots m_{6}} g_{p q} K^{n p I J} K^{q K L}\left(u_{i j}{ }^{I J}+v_{i j I J}\right)\left(u^{i j} K L+v^{i j K L}\right) \text {. }
$$

Here, I suppressed the explicit dependence on the coordinates.

The r.h.s. of eqs. (3.11), (3.12) further simplifies using the uplift Ansatz for the inverse metric in eq. (3.2) and the definition of the Killing two-form in eq. (A.15). It is proportional to

$$
\stackrel{\circ}{D}^{m} \log \Delta=\Delta^{-1} \stackrel{\circ}{D}^{m} \Delta=\frac{1}{2} g^{p q} \stackrel{\circ}{D}^{m} g_{p q},
$$

which finally gives a simpler non-linear Ansatz for the six-form potential, i.e.

$$
\begin{aligned}
& \Delta A^{m}(x, y)+3 \sqrt{2} \stackrel{\zeta}{\zeta}^{m}(y)=\frac{9 \sqrt{2}}{4 m_{7}} \stackrel{\circ}{D}^{m} \log \Delta(x, y),
\end{aligned}
$$

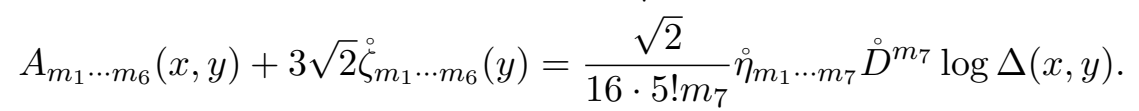

This result has already been derived in [26]. In comparison to eqs. (3.11), (3.12), the Ansätze in eqs. (3.14), (3.15) do not contain the metric $g_{m n}$. However, they require an explicit expression for the warp factor, which also can only be given in particular cases.

\section{New non-linear Ansätze for the metric $g_{m n}$, the warp factor $\Delta$ and the full internal three-form potential $A_{m n p}$}

In this section, I derive a new non-linear metric Ansatz for the uplift of $\mathrm{SO}(8)$ gauged $N=8$ supergravity to 11 -dimensional supergravity. In combination with the expressions for the inverse metric and the three-form with mixed index structure in eqs. (3.2), (3.4), I find further uplift Ansätze for the warp factor and the internal three-form potential $A_{m n p}$. Note that recent work derived similar coordinate-free formulas (eqs. (24-26) of [20]) in a different form.

Following the strategy of the previous section, I consider the relation

$$
\mathcal{V}_{m p A B} \mathcal{V}^{p 8}{ }_{C D} \mathcal{V}_{n q}{ }^{[A B} \mathcal{V}^{q 8 C D]}=\mathcal{V}_{m p i j} \mathcal{V}^{p 8}{ }_{k l} \mathcal{V}_{n q}{ }^{[i j} \mathcal{V}^{q 8 k l]}
$$

Let us use eqs. (2.14), (2.15) on the l.h.s.: all terms including a factor of $A_{m n p}$ are of the form

$$
\ldots A_{m n p} \Gamma_{[A B}^{n} \Gamma_{C D]}^{p} \cdots=0
$$

but such expressions vanish because an antisymmetric index pair $[n p]$ is contracted with a symmetric index pair $(n p)$.

One finally computes the traces of the $\Gamma$-matrices using eq. (A.32) and finds that the l.h.s. of eq. (4.1) is proportional to the metric $g_{m n}$,

$$
\Delta^{-2} g_{m n}=\frac{16}{3} \mathcal{V}_{m p i j} \mathcal{V}^{p}{ }_{k l} \mathcal{V}_{n q}{ }^{[i j} \mathcal{V}^{q k l]}
$$


For the r.h.s., I use eqs. (2.29), (2.30) and find that

$$
\mathcal{V}_{m p[i j} \mathcal{V}^{p}{ }_{k l]}=-\frac{i}{32} K_{m p}{ }^{I J} K^{p K L}\left(u_{[i j}^{I J}-v_{[i j I J}\right)\left(u_{k l]}{ }^{K L}+v_{k l] K L}\right) .
$$

For some readers, eqs. (4.3), (4.4) together already represent a useful metric Ansatz in terms of the Killing forms and the four dimensional scalar fields. However, one may simplify the resulting expression further: using eqs. (A.26), (A.30) in appendix A yields

$$
\mathcal{V}_{m p[i j} \mathcal{V}^{p}{ }_{k l]}=-\frac{i}{8}\left(\mathcal{A}_{m i j k l}-\mathcal{B}_{m i j k l}\right),
$$

where I defined the convenient tensors

$$
\begin{aligned}
\mathcal{A}_{m i j k l}(x, y) & =\frac{1}{4} K_{m n}{ }^{[I J}(y) K^{n K L]}(y)\left(u_{i j}{ }^{I J} u_{k l}{ }^{K L}-v_{i j I J} v_{k l K L}\right)(x), \\
\mathcal{B}_{m i j k l}(x, y) & =K_{m}{ }^{I J}(y)\left(u_{i j}{ }^{I K} v_{k l J K}-v_{i j I K} u_{k l}{ }^{J K}\right)(x) .
\end{aligned}
$$

By definition, these are totally antisymmetric in the SU(8) indices $[i j k l]$ and depend on all 11 coordinates $(x, y)$. Note that a certain linear combination of both tensors is equal to the 'non-metricity' $\mathcal{P}_{m i j k l}$ in the $\mathrm{SO}(8)$ invariant vacuum $[9,26] .{ }^{7}$ One finally finds the metric Ansatz in terms of these tensors, i.e.

$$
\Delta^{-2} g_{m n}(x, y)=\frac{1}{12}\left(\mathcal{A}_{m i j k l}-\mathcal{B}_{m i j k l}\right)\left(\mathcal{A}_{n}^{i j k l}-\mathcal{B}_{n}{ }^{i j k l}\right)(x, y) .
$$

This Ansatz is quartic in the four-dimensional scalar fields $u_{i j}^{I J}$ and $v_{i j} I J$, whereas the Ansätze for the inverse metric and the mixed three-form potential were only quadratic.

Let us combine the Ansätze for the metric and the inverse metric in eqs. (4.8), (3.2) to get a new Ansatz for the warp factor. This can be done because the new metric Ansatz contains a proportionality factor of $\Delta^{-2}$. One finds

$$
\Delta^{-3}(x, y)=\frac{1}{28 \cdot 4 !} \mathcal{C}_{i j}{ }^{k l m n}(x, y) \mathcal{C}^{i j}{ }_{k l m n}(x, y)
$$

where the tensor $\mathcal{C}_{p q}{ }^{i j k l}$ is defined as

$$
\mathcal{C}_{p q}^{i j k l}(x, y)=K^{m I J}(y)\left(u_{p q}^{I J}+v_{p q I J}\right)(x)\left(\mathcal{A}_{m}^{i j k l}-\mathcal{B}_{m}^{i j k l}\right)(x, y) .
$$

Similarly, one combines the Ansatz for the three-form with mixed index structure in eq. (3.4) with the metric Ansatz in eq. (4.8) to obtain a new Ansatz for the full internal three-form potential, i.e.

$\Delta^{-3} A_{m n p}(x, y)=-\frac{\sqrt{2} i}{48 \cdot 4 !} K_{m n}{ }^{I J}(y)\left(u^{i j}{ }_{I J}-v^{i j I J}\right)(x) \mathcal{C}_{i j}{ }^{q r s t}(x, y)\left(\mathcal{A}_{p q r s t}-\mathcal{B}_{p q r s t}\right)(x, y)$.

The new Ansätze for the warp factor and the three-form potential are sextic in the scalar fields $u_{i j}^{I J}$ and $v_{i j} I J$.

\footnotetext{
${ }^{7}$ In [9], the non-metricity $\mathcal{P}_{m i j k l}$ was denoted by $\mathcal{A}_{m i j k l}$.
} 
It may still be possible to simplify the new Ansätze using some $\mathrm{E}_{7(7)}$ properties of the $u_{i j}{ }^{I J}$ and $v_{i j I J}$ tensors $[4,9]$. One such simplification concerns the $C_{p q}{ }^{i j k l}$ tensor that occurs in both, the warp factor and the three-form potential. For the rest of this section, I show that it factorizes into ${ }^{8}$

$$
\mathcal{C}_{p q}{ }^{i j k l}(x, y)=\frac{4}{3} \delta^{[i}{ }_{[p}\left(\mathcal{C}_{1 q]}{ }^{j k l]}(x, y)+2 \mathcal{C}_{2 q]}{ }^{j k l]}(x, y)-2 T_{q]}{ }^{j k l]}(x)\right),
$$

where

$$
\begin{aligned}
\mathcal{C}_{1 p}{ }^{i j k}(x, y)= & K^{I J K L}(y)\left(u_{I J}^{j k}+v^{j k I J}\right)\left(u^{i m}{ }_{K M} u_{p m}{ }^{L M}-v^{i m K M} v_{p m L M}\right)(x), \\
\mathcal{C}_{2 p}{ }^{i j k}(x, y)= & K^{I J K L}(y)\left(u_{I M}^{j k}+v^{j k I M}\right)\left[\left(u^{i m}{ }_{[J K} v_{p m L M]}-v^{i m[J K} u_{p m}{ }^{L M]}\right)\right. \\
& \left.-\frac{1}{8} \delta_{p}^{i}\left(u^{m n}{ }_{[J K} v_{m n L M]}-v^{m n[J K} u_{m n}{ }^{L M]}\right)\right](x) .
\end{aligned}
$$

The selfdual tensor $K^{I J K L}$ is defined as a certain combination of Killing vectors in eq. (A.38). It satisfies some useful relations given in appendix A. The third term in eq. (4.12) represents the $T$-tensor, which is defined in [4],

$$
T_{i}^{j k l}(x)=\left(u_{I J}^{k l}+v^{k l I J}\right)\left(u_{i m}^{J K} u_{K I}^{j m}-v_{i m J K} v^{j m K I}\right)(x) .
$$

It only depends on spacetime coordinates $x$ and satisfies the property

$$
\left(u_{p q}^{I J}+v_{p q I J}\right)\left(u_{I K}^{i j} v^{k l J K}-v^{i j I K} u_{J K}^{k l}\right)=\frac{4}{3} \delta_{[p}^{[i} T_{q]}{ }^{j k l]} .
$$

For further relations concerning the $T$-tensor, see [4, 9]. Note that the only difference between $C_{1 p}{ }^{i j k}$ and the $T$-tensor is the $K^{I J K L}$-factor in eq. (4.13) instead of a $\delta_{K L}^{I J}$-factor in eq. (4.15). This gives rise to interpret $\mathcal{C}_{1}$ and $\mathcal{C}_{2}$ as the $y$-dependent twins of the $T$-tensor.

In order to prove eq. (4.12), one starts with eq. (4.10) and replaces the tensors $\mathcal{A}_{m}{ }^{i j k l}$ and $\mathcal{B}_{m}{ }^{i j k l}$ with the respective expressions in eqs. (4.6), (4.7). Secondly, using eqs. (A.25), (A.41) gives

$$
\begin{aligned}
\mathcal{C}_{p q}{ }^{i j k l}= & -2 K^{I J K L}\left(u_{p q}{ }^{I M}+v_{p q I M}\right)\left(u^{i j}{ }_{[J K} u^{k l}{ }_{L M]}-v^{i j[J K} v^{k l L M]}\right) \\
& -K^{I J K L}\left(u_{p q}{ }^{K L}+v_{p q K L}\right)\left(u^{i j}{ }_{I M} v^{k l J M}-v^{i j I M} u^{k l}{ }_{J M}\right)-\frac{8}{3} \delta^{[i}{ }_{[p} T_{q]}{ }^{j k l]},
\end{aligned}
$$

which can be rearranged,

$$
\begin{aligned}
\mathcal{C}_{p q}{ }^{i j k l}= & 2 K^{I J K L}\left(u^{[i j}{ }_{I J}+v^{[i j I J}\right)\left(u^{k l]}{ }_{K M} u_{p q}{ }^{L M}-v^{k l] K M} v_{p q L M}\right) \\
& +4 K^{I J K L}\left(u^{[i j}{ }_{I M}+v^{[i j I M}\right)\left(u^{k l]}{ }_{[J K} v_{p q L M]}-v^{k l][J K} u_{p q}{ }^{L M]}\right)-\frac{8}{3} \delta^{[i}{ }_{[p} T_{q]}{ }^{j k l]} .
\end{aligned}
$$

\footnotetext{
${ }^{8}$ I thank Hadi Godazgar for pointing this out.
} 
Finally, I use eq. (4.7) of [4] and eq. (5.21) of [9], i.e.

$$
\begin{aligned}
& \left.\left(u_{I M}^{i j} u_{k l}^{J M}-v^{i j I M} v_{k l J M}\right)\right|_{[I J]}=\left.\frac{2}{3} \delta_{[k}^{[i}\left(u_{I M] m}^{j M} u_{l] m}{ }^{J M}-v^{j] m I M} v_{l] m J M}\right)\right|_{[I J]} \\
& \left.\left(u^{i j}{ }_{I J} v_{k l K L}-v^{i j I J} u_{k l}{ }^{K L}\right)\right|_{[I J K L]^{+}}=\left.\frac{2}{3} \delta_{[k}^{[i}\left(u^{j] m}{ }_{I J} v_{l] m K L}-v^{j] m I J} u_{l] m}{ }^{K L}\right)\right|_{[I J K L]^{+}} \\
& -\left.\frac{1}{12} \delta_{k l}^{i j}\left(u_{I J}^{m n} v_{m n K L}-v^{m n I J} u_{m n} K L\right)\right|_{[I J K L]^{+}},
\end{aligned}
$$

where $\left.\right|_{[I J K L]}+$ represents the projection onto the selfdual part. This completes the proof of eq. (4.12). In order to keep the formulas short, I do not insert the factorization of the $\mathcal{C}_{p q}{ }^{i j k l}$ tensor into the uplift Ansätze for the warp factor and the three-form. However, one should always keep in mind that these expressions can still be simplified by eq. (4.12).

I must emphasize that the antisymmetry of the three-form potential $A_{m n p}$ is not apparent from the new Ansatz in eq. (4.11). This may be a hint that it still can be simplified using the $\mathrm{E}_{7(7)}$ properties of the $u_{i j}{ }^{I J}$ and $v_{i j I J}$ tensors. One should check such a simplification in future work. Note that the recent three-form Ansatz in [20] is given in a coordinate-free form, hence its components are fully antisymmetric by definition.

In section 6, I will test the new metric Ansatz for the $G_{2}$ invariant solution of 11dimensional supergravity. Note that the Ansätze for the warp factor and the flux originate from the old formulas for $\Delta^{-1} g^{m n}$ and $A_{m n}{ }^{p}$ using the new metric Ansatz. Since these old expressions were already tested for a $\mathrm{G}_{2}$ invariant solution [16], I do not re-check eqs. (4.9), (4.11) explicitly. For a consistent test, it will be sufficient to compute the metric by eq. (4.8) and compare it with the existing expression in [16]. ${ }^{9}$

\section{A new non-linear Ansatz for the four-form field-strength}

In this section, I present a new non-linear Ansatz for the four-form field-strength

$$
F_{m n p q}=4 ! \stackrel{\circ}{D}_{[m} A_{n p q]} .
$$

So far, the internal three-form potential was only known in particular cases and it was yet very complicated to compute the derivative of an explicit expression for $A_{m n p}$. However, I found a new general uplift Ansatz for $A_{m n p}$ in the previous section. In particular, at the level of 11-dimensional vielbein components (eqs. (3.3), (4.1)), one finds

$$
A_{m n p}=\frac{16 \sqrt{2}}{9} \Delta^{3} \mathcal{V}_{m n}{ }^{A B} \mathcal{V}_{p q}{ }^{[C D} \mathcal{V}^{q E F]} \mathcal{V}^{r}{ }_{A B} \mathcal{V}_{r s} C D \mathcal{V}^{s}{ }_{E F}
$$

With a look at eqs. (2.14), (2.15) and using eq. (A.22), one has

$$
\mathcal{V}_{p q}{ }^{[C D} \mathcal{V}^{q E F]}=\frac{1}{2}\left(\mathcal{V}_{p q}{ }^{C D} \mathcal{V}^{q E F}+\mathcal{V}_{p q}{ }^{E F} \mathcal{V}^{q C D}\right)
$$

\footnotetext{
${ }^{9}$ Within a $\mathrm{G}_{2}$ invariant solution, an expression for the metric has been found by inverting $\Delta^{-1} g^{m n}$ 'by hand'.
} 
Furthermore, since all SU(8) indices in eq. (5.2) are fully contracted, I can replace the 11-dimensional vielbeine by the four dimensional expressions in eqs. (2.29)-(2.32). This finally yields a general expression for the four-form field-strength, i.e.

$$
F_{m n p q}=\frac{64 \sqrt{2}}{3} \stackrel{\circ}{D}_{[m}\left(\Delta^{3} \mathcal{V}_{n p}{ }^{i_{1} i_{2}}\left(\mathcal{V}_{q] r}{ }^{i_{3} i_{4}} \mathcal{V}^{r i_{5} i_{6}}+\mathcal{V}_{q] r}{ }^{i_{5} i_{6}} \mathcal{V}^{r i_{3} i_{4}}\right) \mathcal{V}^{s}{ }_{i_{1} i_{2}} \mathcal{V}_{s t i_{3} i_{4}} \mathcal{V}_{i_{5} i_{6}}\right)
$$

One can now evaluate the derivative in general. First, one has

$$
\stackrel{\circ}{D}_{m} \Delta^{3}=3 \Delta^{3} \stackrel{\circ}{D}_{m} \log \Delta
$$

hence, one term in $F_{m n p q}$ will be proportional to $A_{[m n p} \stackrel{\circ}{D}_{q]} \log \Delta$. Secondly, the covariant background derivative $\stackrel{D}{m}_{m}$ only acts on the $y$-dependent fields in the vielbein components: the Killing forms and the dual volume potential $\stackrel{\zeta}{\zeta}^{m}$. It does not act on the scalars $u_{i j}{ }^{I J}$ and $v_{i j} I J$. In general,

$$
\begin{aligned}
& \stackrel{\circ}{D}_{m} \mathcal{V}^{n}{ }_{i j}=m_{7} \stackrel{\circ}{g}_{m p}\left(2 \stackrel{\circ}{\zeta}^{[n} \mathcal{V}^{p]}{ }_{i j}-\mathcal{V}^{n p}{ }_{i j}\right) \text {, } \\
& \stackrel{\circ}{D}_{m} \mathcal{V}_{n p i j}=2 m_{7} \stackrel{\circ}{g}_{m[n}\left(-\mathcal{V}_{p] i j}+\stackrel{\circ}{\zeta}^{q} \mathcal{V}_{p] q i j}\right) \text {, } \\
& \stackrel{\circ}{D}_{m} \mathcal{V}^{n p}{ }_{i j}=-2 m_{7}\left(\delta_{m}{ }^{[n}+\stackrel{\circ}{\zeta}_{m} \stackrel{\circ}{\zeta}^{[n}-\stackrel{\circ}{D}_{m} \stackrel{\circ}{\zeta}^{[n}\right) \mathcal{V}^{p]}{ }_{i j}-2 m_{7} \stackrel{\circ}{g}_{m q} \stackrel{\circ}{\zeta}^{[n} \mathcal{V}^{p] q}{ }_{i j},
\end{aligned}
$$

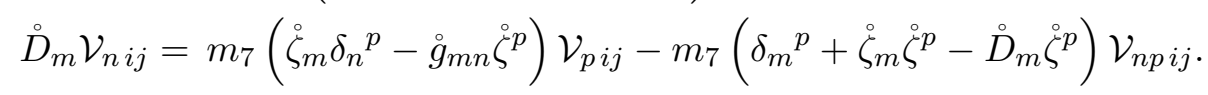

Putting all this together, the resulting intermediate expression for $F_{m n p q}$ becomes rather long and I do not display it here. However, it should be clear that it contains the tensors $\stackrel{\circ}{g}_{m n}, \check{\zeta}^{m}$ as well as all four-dimensional vielbeine $\mathcal{V}^{\mathcal{M}}$ ij. The $\mathrm{SU}(8)$ indices $i j \ldots$ are fully contracted in pairs. I can therefore replace the $\mathcal{V}^{\mathcal{M}}$ ij's by the 11-dimensional vielbein components $\mathcal{V}^{\mathcal{M}}{ }_{A B}$. The final step is to use eqs. (2.14)-(2.17), which introduces the 11-dimensional fields (e.g. $A_{m n p}$ and $A_{m_{1} \cdots m_{6}}$ ) as well as $\Gamma$-matrices. Using eqs. (A.11) for the traces of products of $\Gamma$-matrices, I finally obtain

$$
\begin{aligned}
F_{m n p q}= & -72 A_{[m n p} \stackrel{\circ}{D}_{q]} \log \Delta+\frac{24}{\sqrt{2}} m_{7} A_{[m n p} \stackrel{\circ}{q}_{q] r}\left(\Delta A^{r}+3 \sqrt{2} \stackrel{\circ}{\zeta}^{r}\right) \\
& +\left.\left[4 m_{7}{\stackrel{\circ}{g m r_{1}}}^{r^{r}} \cdots r_{7}\left(g_{n r_{2}} g_{p r_{3}} g_{q r_{4}}-18 A_{n p r_{2}} A_{q r_{3} r_{4}}\right) A_{r_{5} r_{6} r_{7}}\right]\right|_{[m n p q]},
\end{aligned}
$$

where $\left.\right|_{[m n p q]}$ denotes antisymmetrized indices $m n p q$. One eliminates the second term by eq. (3.14),

$$
\begin{aligned}
F_{m n p q}= & -18 A_{[m n p} \stackrel{\circ}{D}_{q]} \log \Delta \\
& +\left.\left[4 m_{7} \stackrel{\circ}{m}_{m r_{1}} \stackrel{\circ}{\eta}^{r_{1} \cdots r_{7}}\left(g_{n r_{2}} g_{p r_{3}} g_{q r_{4}}-18 A_{n p r_{2}} A_{q r_{3} r_{4}}\right) A_{r_{5} r_{6} r_{7}}\right]\right|_{[m n p q]}
\end{aligned}
$$

For some readers, this expression is already in a desired form. However, one can further simplify this expression. First, the term proportional to $\stackrel{\circ}{ }^{r_{1} \cdots r_{7}} A_{q r_{3} r_{4}} A_{r_{5} r_{6} r_{7}}$ can be replaced using eq. (3.9). Together with eq. (3.14), this cancels the term proportional to $\stackrel{\circ}{D}_{m} \log \Delta$. Finally, one turns the tensor density $\stackrel{\circ}{\eta}^{r_{1} \cdots r_{7}}$ into the tensor $\epsilon^{r_{1} \cdots r_{7}}$ (eq. (3.6)) and obtains

$$
\begin{aligned}
F_{m n p q}= & m_{7} \Delta \stackrel{\circ}{g}_{s[m}\left(4 \epsilon_{n p q] r_{1} r_{2} r_{3}} A^{r_{1} r_{2} r_{3}}\right. \\
& \left.-3 g_{n|t|} A_{p q] r} K^{r s I J} K^{t K L}\left(u_{i j}^{I J}+v_{i j I J}\right)\left(u^{i j} K L+v^{i j K L}\right)\right) .
\end{aligned}
$$


This formula appears to be more feasible for practical tests than previous expressions $[20,26]$.

It is not difficult to raise all indices with the inverse metric $g^{m n}$. Therefore, one must keep in mind that the indices of the Killing forms and $\stackrel{\circ}{g n}_{m n}$ are raised with the background metric. All other tensors in eq. (5.12) are covariant, hence

$$
\begin{aligned}
F^{m n p q}= & m_{7} \Delta \stackrel{\circ}{g}_{s t} g^{t[m}\left(4 \epsilon^{n p q] r_{1} r_{2} r_{3} s} A_{r_{1} r_{2} r_{3}}\right. \\
& \left.-3 A^{n p}{ }_{r} K^{q] I J} K^{r s K L}\left(u^{i j}{ }_{I J}+v^{i j I J}\right)\left(u_{i j}{ }^{K L}+v_{i j K L}\right)\right) .
\end{aligned}
$$

Note the power of the last step: until now, the field-strength with upper indices has always been found by raising each lower index of $F_{m n p q}$ with the explicit expression for the inverse metric $g^{m n}$. This was one of the hardest tasks in verifying the $\mathrm{SO}(3) \times \mathrm{SO}(3)$ invariant solution of 11-dimensional supergravity. With the new Ansatz above, it is much simpler to find $F^{m n p q}$. For maximally symmetric spacetimes, these results may also be used to calculate the Ricci tensor using the Einstein equations.

In the next section, I will test the new Ansatz for the four-form field-strength for the $\mathrm{G}_{2}$ invariant solution of 11-dimensional supergravity.

\section{Testing the new uplift Ansätze}

This section presents an essential part of this work: I test the new non-linear Ansätze for the metric $g_{m n}$ and the four-form field-strength $F_{m n p q}$ within a $\mathrm{G}_{2}$ invariant solution of 11-dimensional supergravity. In such a setup, the Ansätze for the inverse metric $\Delta^{-1} g^{m n}$ (eq. (3.2)) and the three-form with mixed index structure (eq. (3.4)) were already checked successfully [16]. The same reference computes the warp factor by taking the determinant of the expression for $\Delta^{-1} g^{m n}$ and the metric $g_{m n}$ by inverting $g^{m n}$. Finally, it calculates the full internal three-form potential $A_{m n p}$ by lowering the third index with the explicit expression for $g_{m n}$. It should be clear that a successful test for the metric Ansatz in eq. (4.8) includes the tests of the Ansätze for the warp factor and the three-form potential in eqs. (4.9), (4.11), since these result from combining the old known Ansätze with the new metric Ansatz.

Here, I compute the metric $\Delta^{-2} g_{m n}$ by eqs. (4.3), (4.4), which is equivalent to use eq. (4.8). I follow the strategy of [16]: one first brings the $\mathrm{E}_{7(7)}$-matrix that encodes the four-dimensional scalars

$$
\mathcal{V}=\left(\begin{array}{ll}
u_{i j}^{I J} & v_{i j I J} \\
v^{i j I J} & u_{I J}^{i j}
\end{array}\right)
$$

into unitary gauge,

$$
\mathcal{V}=\exp \left(\begin{array}{cc}
0 & \phi_{I J K L} \\
\phi^{I J K L} & 0
\end{array}\right),
$$

where $\phi_{I J K L}$ denotes the scalar vacuum expectation value. In this gauge, there is no distinction between $\mathrm{SU}(8)$ indices $i j \ldots$ and $\mathrm{SL}(8, \mathbb{R})$ indices $I J \ldots$ This allows us to write 
the scalar fields $u_{i j}^{I J}$ and $v_{i j I J}$ in terms of the vacuum expectation value $\phi_{I J K L}$. For a $\mathrm{G}_{2}$ invariant configuration, the latter takes the general form,

$$
\phi_{I J K L}(x)=\frac{\lambda(x)}{2}\left(C_{+}^{I J K L} \cos \alpha(x)+i C_{-}^{I J K L} \sin \alpha(x)\right),
$$

where $C_{+}^{I J K L}$ is selfdual and $C_{-}^{I J K L}$ is anti-selfdual. The above expression also defines a scalar field $\lambda(x)$ and a rotation angle $\alpha(x)$. Using the explicit form of the vacuum expectation value in eq. (6.3), one finds the four-dimensional scalars $u_{i j}{ }^{I J}$ and $v_{i j} J J$ in terms of the $\mathrm{G}_{2}$ invariants $C_{ \pm}^{I J K L}$, i.e.

$$
\begin{aligned}
u_{I J}{ }^{K L}= & p^{3} \delta_{K L}^{I J}+\frac{1}{2} p q^{2} \cos ^{2} \alpha C_{+}^{I J K L}-\frac{1}{2} p q^{2} \sin ^{2} \alpha C_{-}^{I J K L}-\frac{i}{8} p q^{2} \sin 2 \alpha D_{-}^{I J K L}, \\
v_{I J K L}= & q^{3}\left(\cos ^{3} \alpha-i \sin ^{3} \alpha\right) \delta_{K L}^{I J}+\frac{1}{2} p^{2} q \cos \alpha C_{+}^{I J K L}+\frac{i}{2} p^{2} q \sin \alpha C_{-}^{I J K L} \\
& -\frac{1}{8} q^{3} \sin 2 \alpha(\sin \alpha-i \cos \alpha) D_{+}^{I J K L},
\end{aligned}
$$

where $p=\cosh \lambda$ and $q=\sinh \lambda$. The tensors $D_{ \pm}^{I J K L}$ are defined as

$$
D_{ \pm}^{I J K L}=\frac{1}{2}\left(C_{+}^{I J M N} C_{-}^{M N K L} \pm C_{-}^{I J M N} C_{+}^{M N K L}\right) .
$$

One now expands the $C_{ \pm}^{I J K L}$ tensors into the (anti-)selfdual bases provided by the Killing forms defined in eq. (A.19), (A.20),

$$
\begin{aligned}
C_{+}^{I J K L} & =\frac{\xi}{6} K_{m}{ }^{[I J} K^{m K L]}+\frac{1}{12} \xi^{m} K_{m n}{ }^{[I J} K^{n K L]}-\frac{3}{2} K_{m}{ }^{[I J} K_{n}{ }^{K L]} \xi^{m n}, \\
C_{-}^{I J K L} & =\frac{1}{2} S^{m n p} K_{m n}{ }^{[I J} K_{p}{ }^{K L]} .
\end{aligned}
$$

The occurring components $\xi, \xi^{m}, \xi^{m n}$ and $S^{m n p}$ are $\mathrm{SO}(7)$ tensors ${ }^{10}$ on the round $S^{7}$, hence, its indices are raised and lowered with the background metric $\stackrel{\circ}{g}_{m n}$. Note that $S^{m n p}$ is totally antisymmetric by construction. Furthermore, one finds the useful relations [16]

$$
\begin{aligned}
& \xi^{m n} \stackrel{\circ}{g}_{m n}=\xi, \quad \xi_{m} \xi_{n}=\left(9-\xi^{2}\right) \stackrel{\circ}{g}_{m n}-6(3-\xi) \xi_{m n}, \quad \xi_{m} \xi^{m}=(21+\xi)(3-\xi), \\
& S^{m n r} S_{p q r}=2 \delta_{p q}^{m n}+\frac{1}{6} \eta^{m n}{ }_{p q r s t} S^{r s t}, \quad S^{[m n p} S^{q] r s}=\frac{1}{4} \eta^{m n p q[r}{ }_{t u} S^{s] t u}, \\
& S^{m[n p} S^{q r] s}=\frac{1}{6} \eta^{n p q r(m}{ }_{t u} S^{s) t u} .
\end{aligned}
$$

From the decomposition of the $C_{ \pm}^{I J K L}$ tensors in eqs. (6.7), (6.8), one finds the useful contractions

$$
\begin{aligned}
& C_{+}^{I J K L} K_{m}{ }^{K L}=-2 \xi_{m n} K^{n I J}-\frac{1}{3} \xi^{n} K_{m n}{ }^{I J}, \\
& C_{+}^{I J K L} K_{m n}{ }^{K L}=\frac{2}{3} \xi_{[m} K_{n]}{ }^{I J}+\left(\frac{2}{3} \xi \delta_{m n}^{p q}-4 \delta_{[m}{ }^{p} \xi_{n]}{ }^{q}\right) K_{p q}^{I J}, \\
& C_{-}^{I J K L} K_{m}{ }^{K L}=S_{m n p} K^{n p I J}, \\
& C_{-}^{I J K L} K_{m n}{ }^{K L}=2 S_{m n p} K^{p I J}-\frac{1}{6}{\stackrel{\ominus}{m n p_{1} \cdots p_{5}}} S^{p_{1} p_{2} p_{3}} K^{p_{4} p_{5} I J},
\end{aligned}
$$

\footnotetext{
${ }^{10}$ In [16], $S^{m n p}$ was denoted by $\stackrel{S}{m n p}^{m}$.
} 
as well as for the $D_{ \pm}^{I J K L}$ tensors,

$$
\begin{aligned}
& D_{+}^{I J K L} K_{m}{ }^{K L}=\left(\frac{\xi}{3} S_{m n p}-\xi_{m}{ }^{q} S_{n p q}-2 S_{m n q} \xi_{p}^{q}+\frac{1}{36} \stackrel{\circ}{\eta}_{m n p q r s t} \xi^{q} S^{r s t}\right) K^{n p I J}, \\
& D_{+}^{I J K L} K_{m n}{ }^{K L}=\left(\frac{2}{3} \xi S_{m n p}-4 \xi_{[m}{ }^{q} S_{n] p q}-2 S_{m n q} \xi_{p}{ }^{q}+\frac{1}{18} \stackrel{\circ}{\eta}_{m n p q r s t} \xi^{q} S^{r s t}\right) K^{p I J}+ \\
& +\frac{1}{3}\left(\xi_{[m} S_{n] p q}-S_{m n p} \xi_{q}-\frac{\xi}{3} \grave{\eta}_{m n p q r s t} S^{r s t}\right. \\
& \left.+\stackrel{\circ}{\text { mnprstu }}_{\xi_{q}}^{r} S^{s t u}+{\stackrel{\circ}{\eta_{[m \mid p q r s t u}}}_{n}{ }^{r} S^{s t u}\right) K^{p q I J} \\
& D_{-}^{I J K L} K_{m}^{K L}=\frac{2}{3} S_{m n p} \xi^{n} K^{p I J} \\
& +\left(\frac{\xi}{3} S_{m n p}+\xi_{m}{ }^{q} S_{n p q}-2 S_{m n q} \xi_{p}^{q}-\frac{1}{36} \dot{\eta}_{m n p q r s t} \xi^{q} S^{r s t}\right) K^{n p I J}, \\
& D_{-}^{I J K L} K_{m n}{ }^{K L}=\left(-\frac{2}{3} \xi S_{m n p}+4 \xi_{[m}{ }^{q} S_{n] p q}-2 S_{m n q} \xi_{p}{ }^{q}+\frac{1}{18} \grave{\eta}_{m n p q r s t} \xi^{q} S^{r s t}\right) K^{p I J} \\
& +\frac{1}{3}\left(-\xi_{[m} S_{n] p q}-S_{m n p} \xi_{q}+\stackrel{\circ}{\eta}_{m n p r s t u} \xi_{q}^{r} S^{s t u}-\stackrel{\circ}{\eta}_{[m \mid p q r s t u} \xi_{n]}{ }^{r} S^{s t u}\right) K^{p q I J} .
\end{aligned}
$$

Now, I write the metric $g_{m n}$ in terms of the components $\xi, \xi^{m}, \xi^{m n}$ and $S^{m n p}$ defined above. Therefore, one first computes $\mathcal{V}_{m p[i j} \mathcal{V}_{k l]}^{p}$ (or better: $\mathcal{V}_{m p[I J} \mathcal{V}^{p}{ }_{K L]}$ ) using eq. (4.4) and expands the scalar fields $u_{I J}{ }^{K L}$ and $v_{I J K L}$ in terms of the $C_{ \pm}^{I J K L}$ and $D_{ \pm}^{I J K L}$ tensors (eqs. (6.4), (6.5)). Secondly, one uses the contractions above together with eqs. (A.27), (A.28) in appendix A to bring $\mathcal{V}_{m p[I J} \mathcal{V}^{p}{ }_{K L]}$ into the basis provided by eqs. (A.19), (A.20),

$$
\begin{aligned}
\mathcal{V}_{m p[I J} \mathcal{V}_{K L]}^{p}= & a_{m} K_{n}{ }^{[I J} K^{n K L]}+b_{m}{ }^{n} K_{n p}{ }^{[I J} K^{p K L]} \\
& +c_{m}{ }^{n p} K_{n}{ }^{[I J} K_{p}{ }^{K L]}+d_{m}{ }^{n p q} K_{[n p}{ }^{[I J} K_{q]}{ }^{K L]} .
\end{aligned}
$$

The respective coefficients $a_{m}, b_{m}{ }^{n}, c_{m}{ }^{n p}$ and $d_{m}{ }^{n p q}$ are rather long expressions and I do not display them here. However, it should be clear that they only depend on the $\mathrm{SO}(7)$ tensors $\xi, \xi^{m}, \xi^{m n}$ and $S^{m n p}$. Finally, one computes the metric via eq. (4.3). For the contractions of the indices $I J K L$, one uses eqs. (A.34)-(A.37) and for the contractions of the $\mathrm{SO}(7)$ indices, one uses the identities in eqs. (6.9), (6.10). This finally results in

$$
\Delta^{-2} g_{m n}=b_{0}\left[\left(b_{0}+3 c v s\right) \stackrel{\circ}{g}_{m n}+\operatorname{cvs} \xi_{m n}\right],
$$

where I made the following definitions:

$$
c=\cosh 2 \lambda, \quad s=\sinh 2 \lambda, \quad v=\cos \alpha, \quad b_{0}=c^{2}+v^{2} s^{2}-\frac{9+\xi}{6} \text { cvs } .
$$

The test for the inverse metric Ansatz (eq. (3.2)) was already performed in [16]. The corresponding final expression is

$$
\Delta^{-1} g^{m n}=\left(c^{3}+v^{3} s^{3}\right) \stackrel{\circ}{g}^{m n}-c v s(c+v s) \xi^{m n} .
$$


Combining the explicit expressions for the metric and its inverse in eqs. (6.20), (6.22) and using the identities in eqs. (6.9), (6.10), one finds that

$$
\Delta^{-2} g_{m p} \Delta^{-1} g^{p n}=b_{0}^{2}(c+v s)^{3} \delta_{m}^{n} .
$$

This is exactly the combination of the metric and its inverse that defines the warp factor in eq. (4.9), hence

$$
\Delta^{-3}=b_{0}^{2}(c+v s)^{3} .
$$

The explicit expressions for the metric and the warp factor in eqs. (6.20), (6.24) reproduce the results of [16]. The reader may also check that the determinant of the metric in eq. (6.20) indeed, reproduces eq. (6.24). The test is hence, successful.

For the remaining test of the field-strength Ansatz in eq. (5.12), I use the explicit expression for $A_{m n p}$ that was found in [16],

$$
\begin{aligned}
A_{m n p}= & \frac{\sqrt{2} \tan \alpha}{72 b_{0}} \frac{v s}{c+v s}\left(9 v s(c-v s) \xi_{[m}{ }^{q} S_{n p] q}\right. \\
& \left.+\frac{1}{12} v s(c+v s) \dot{\circ}_{m n p q r s t} \xi^{q} S^{r s t}+(2 c-v s)(3 c-\xi v s) S_{m n p}\right) .
\end{aligned}
$$

Note that this expression is slightly simplified using the identities in eqs. (6.9), (6.10). Furthermore, the formula for $A_{m n p}$ above differs from the expression given in [16] by a factor of $1 / 6$, which is due to my conventions. However, the definition of the field-strength in eq. (5.1) differs from the corresponding definition in [16] by a factor of 6 . Hence, the new Ansatz for $F_{m n p q}$ in eq. (5.12) should give the same expression as already computed in [16] by calculating the derivative of eq. (6.25) directly.

A convenient way to use the new Ansatz is

$$
\begin{aligned}
& F_{m n p q}=\left[4 m_{7} \Delta^{6} \stackrel{\circ}{g}_{m r_{1}}\left(\Delta^{-2} g_{n r_{2}}\right)\left(\Delta^{-2} g_{p r_{3}}\right)\left(\Delta^{-2} g_{q r_{4}}\right) \stackrel{\circ}{\eta}^{r_{1} \cdots r_{7}} A_{r_{5} r_{6} r_{7}}-\right. \\
& \left.-3 m_{7} \Delta^{3}\left(\Delta^{-2} g_{n t}\right) A_{p q r} K^{r s I J} K^{t K L}\left(u_{i j}^{I J}+v_{i j I J}\right)\left(u^{i j} K L+v^{i j K L}\right)\right]\left.\right|_{[m n p q]},
\end{aligned}
$$

such that one may use eqs. (6.20), (6.24), (6.25) directly. For the term involving the Killing forms and the four-dimensional scalars, I follow the same strategy as described earlier in this section. I find

$$
\begin{aligned}
& K^{m n I J} K^{p K L}\left(u_{i j}{ }^{I J}+v_{i j I J}\right)\left(u_{K L}^{i j}+v^{i j K L}\right) \\
& =\frac{8}{3} \operatorname{cvs}(c+v s) \xi^{[m} \stackrel{\circ}{g}^{n] p} \\
& \quad+s^{2} \sin ^{2} \alpha\left[12 v s \xi_{q}^{[m} S^{n p] q}-\frac{1}{9} v s \dot{\eta}^{m n p q r s t} \xi_{q} S_{r s t}-\left(8 c+\frac{4}{3} \xi v s\right) S^{m n p}\right] .
\end{aligned}
$$

Putting all together and using eqs. (6.9), (6.10) finally results in

$$
\begin{aligned}
F_{m n p q}= & \frac{\sqrt{2} v^{2} s^{2} \tan \alpha}{3 b_{0}} m_{7}\left[\frac{c-v s}{v s} \grave{\eta}_{m n p q r s t} S^{r s t}+\left(\frac{2 c-v s}{c+v s}+\frac{c^{2}-v^{2} s^{2}}{b_{0}}\right) \xi_{[m} S_{n p q]}\right. \\
& \left.+\frac{1}{6(3-\xi)}\left(\frac{2 c-v s}{c+v s}-\frac{(c-v s)^{2}}{b_{0}}\right) \xi_{[m} \stackrel{\circ}{n p q] r s t u}^{r} S^{s t u}\right],
\end{aligned}
$$

which matches exactly the expression found in [16]. The test is hence, successful. 


\section{Conclusion}

In this paper, I derive a new non-linear metric Ansatz for the uplift of $N=8$ supergravity to 11-dimensional supergravity. An uplift Ansatz for the inverse metric, scaled with the warp factor, $\Delta^{-1} g^{m n}$, has already been known for a long time [15]. However, inverting this expression in order to find $\Delta g_{m n}$ was only possible in certain cases, for example when the theory is $\mathrm{G}_{2}, \mathrm{SO}(3) \times \mathrm{SO}(3)$ or $\mathrm{SU}(3) \times \mathrm{U}(1) \times \mathrm{U}(1)$ invariant [16-19]. Also the warp factor $\Delta$ could only be extracted by taking the determinant in such particular cases. Following the strategy of [14], I present a new general uplift Ansatz for $\Delta^{-2} g_{m n}$ in terms of the four-dimensional scalar fields and the Killing forms on the background (eqs. (4.3)-(4.8)). Note that this Ansatz is similar to a recent coordinate-free expression [20]. However, the formula presented here seems to be more feasible for practical tests: I tested the new metric Ansatz within a $\mathrm{G}_{2}$ invariant solution of 11-dimensional supergravity in section 6 .

Similarly to [20], the new formula can further be used in order to find non-linear uplift Ansätze for the warp factor and the full internal three-form potential in general. For the warp factor, I combine the old Ansatz for $\Delta^{-1} g^{m n}$ with the new one for $\Delta^{-2} g_{m n}$, which gives a new Ansatz for $\Delta^{-3}$ (eq. (4.9)). Furthermore, I derive a general Ansatz for the full internal three-form potential $A_{m n p}$ (eq. (4.11)) by combining the old flux Ansatz for $A_{m n}{ }^{p}[14]$ with the new metric Ansatz. However, this new formula does not reveal the total antisymmetry of the three-form. This may be a hint that one can further simplify the expression for $A_{m n p}$ using some $\mathrm{E}_{7(7)}$ identities for the four-dimensional scalar fields. I hope that I can provide such a simplification in future work.

In a second part of this paper, I derive a new general non-linear uplift Ansatz for the four-form field-strength $F_{m n p q}$ within the considered uplift of $N=8$ supergravity to 11-dimensional supergravity. So far, the simplest way to derive $F_{m n p q}$ was to compute the derivative of the three-form potential. However, this required an explicit expression for the flux, which is only given in particular cases, e.g. the $\mathrm{G}_{2}, \mathrm{SO}(3) \times \mathrm{SO}(3)$ or $\mathrm{SU}(3) \times \mathrm{U}(1) \times \mathrm{U}(1)$ invariant solutions of 11-dimensional supergravity. With the new Ansatz for the fieldstrength (eq. (5.12)), there is no need to compute derivatives anymore. It is given in terms of the metric, the flux as well as the four-dimensional scalars and background Killing forms. The formula holds in general and also passes a very non-trivial test for a $\mathrm{G}_{2}$ invariant solution of 11-dimensional supergravity.

The new Ansatz for the field-strength also provides a simple expression for $F^{m n p q}$ (eq. (5.13)) in terms of the inverse metric, the flux as well as the four-dimensional scalars and background Killing forms. This new formula makes it redundant to raise each index of $F_{m n p q}$ with the explicit expression for the inverse metric, $g^{m n}$, which was so far, the only way to derive $F^{m n p q}$. The new direct Ansatz for $F^{m n p q}$ is also much more effective than this old method - in order to verify the $\mathrm{SO}(3) \times \mathrm{SO}(3)$ invariant solution of 11-dimensional supergravity, the index-raising of $F_{m n p q}$ was one of the hardest tasks [26].

In future, one may also find new Ansätze for the Christoffel connections in 11dimensional supergravity in terms of the four-dimensional scalars and background Killing forms. Since they are given by the first derivative of the metric, one could find new simple expressions in full analogy to the derivation of the field-strength Ansatz. Similarly, one could derive a non-linear Ansatz for the Riemann tensor. 
In this paper, all Ansätze are derived within the $\mathrm{S}^{7}$ reduction of 11-dimensional supergravity. This leads to the compact gauging $\mathrm{SO}(8)$. However, the methods provided here should also apply in general for other truncations. As a first example, one may extend the theory to the non-compact $\operatorname{CSO}(p, q, r)$ gaugings [27, 28]. In this case, the $I J$ indices of the Killing forms are raised and lowered with the $\operatorname{CSO}(p, q, r)$-metric $\eta_{I J}$ instead of the $\mathrm{SO}(8)$ metric $\delta_{I J}$. This effects the definition of the matrix $\mathcal{R}^{\mathcal{M}}{ }_{\mathcal{N}}$ in eqs. (2.22)-(2.25) and hence, the $\mathcal{A}_{m i j k l}$ and $\mathcal{B}_{m i j k l}$ tensors in eqs. (4.6), (4.7). Thus, the new Ansätze for the metric, the three-form and warp-factor will be slightly modified. However, the new Ansatz for the four-form field-strength will change more dramatically: eqs. (5.6)-(5.9) do not hold if the $I J$ indices of the Killing forms are raised and lowered with the full $\operatorname{CSO}(p, q, r)$ metric. Since the new Ansatz for $F_{m n p q}$ depends on those identities, it will take much more effort to derive an adapted Ansatz for the four-form within the non-compact gaugings. Finally, the presented methods may also be used for the reduction from type IIB supergravity to five dimensions [29-31].

\section{Acknowledgments}

I thank Hermann Nicolai, Axel Kleinschmidt as well as Hadi and Mahdi Godazgar for useful discussions.

\section{A Gamma matrices, Killing spinors, Killing vectors and Killing forms of the $S^{7}$}

One defines a set of euclidean, antisymmetric and purely imaginary $8 \times 8 \Gamma$-matrices $\left(\Gamma^{\dagger}=\Gamma\right)$. These generate the euclidean Clifford algebra in seven dimensions, ${ }^{11}$

$$
\left\{\Gamma_{a}, \Gamma_{b}\right\}=2 \delta_{a b} \mathbb{I}_{8 \times 8}
$$

Let us choose a Majorana representation: the charge conjugation matrix that defines spinor conjugates or raises and lowers spinor indices is set to the unit matrix. Thus, the eight Killing spinors of the round $S^{7}$ satisfy $\bar{\eta}^{I}=\left(\eta^{I}\right)^{\dagger}$. Furthermore, one may choose them to be orthonormal,

$$
\bar{\eta}^{I} \eta^{J}=\delta^{I J}, \quad \eta^{I} \bar{\eta}^{I}=\mathbb{I}_{8 \times 8} .
$$

The flat $\Gamma$-matrices define two types of 'curved' $\Gamma$-matrices: first, matrices $\stackrel{\circ}{\Gamma}_{m}=$ $\stackrel{\circ}{m}_{m}^{a} \Gamma_{a}$ are defined on the round seven-sphere, its indices are raised and lowered with the background metric $\stackrel{\circ}{g}_{m n}$. Secondly, matrices $\Gamma_{m}=e_{m}^{a} \Gamma_{a}$ are defined on the deformed $S^{7}$ and its indices are raised and lowered with the full internal metric $g_{m n}$.

The Killing spinors are defined on the background $S^{7}$ and hence, satisfy

$$
i \stackrel{\circ}{D}_{m} \eta^{I}=\frac{m_{7}}{2} \stackrel{\circ}{\Gamma}_{m} \eta^{I}, \quad-i \stackrel{\circ}{D}_{m} \bar{\eta}^{I}=\frac{m_{7}}{2} \bar{\eta}^{I} \stackrel{\circ}{\Gamma}_{m} .
$$

\footnotetext{
${ }^{11}$ In the following, I suppress the $\mathrm{SU}(8)$ indices that label rows and columns of the matrices, $\Gamma_{a}=\left(\Gamma_{a}\right)_{A B}$.
} 
Here, $\stackrel{\circ}{D}_{m}$ is the covariant derivative with respect to the internal background metric $\stackrel{\circ}{g}_{m n}$ and $m_{7}$ is the inverse $S^{7}$ radius.

The $\Gamma$-matrices can be used to define two sets of $8 \times 8$ matrices,

$$
\stackrel{\circ}{\Gamma}_{m_{1} \ldots m_{i}}=\stackrel{\circ}{\Gamma}_{\left[m_{1}\right.} \ldots \stackrel{\circ}{\Gamma}_{\left.m_{i}\right]}, \quad \quad \Gamma_{m_{1} \ldots m_{i}}=\Gamma_{\left[m_{1}\right.} \ldots \Gamma_{\left.m_{i}\right]}
$$

for $i=2, \ldots, 7$. For example,

$$
\Gamma_{m n p}=\frac{1}{3 !}\left(\Gamma_{m n p}+\Gamma_{n p m}+\Gamma_{p m n}-\Gamma_{m p n}-\Gamma_{n m p}-\Gamma_{p n m}\right) .
$$

$\Gamma$-matrices with one and two indices are antisymmetric and $\Gamma$-matrices with three indices are symmetric. The two sets $\left(\mathbb{I}_{8 \times 8}, \stackrel{\circ}{\Gamma}_{m}, \stackrel{\circ}{\Gamma}_{m n}, \stackrel{\circ}{\Gamma}_{m n p}\right)$ and $\left(\mathbb{I}_{8 \times 8}, \Gamma_{m}, \Gamma_{m n}, \Gamma_{m n p}\right)$ each contain $1+7+21+35=64$ independent matrices. Hence, they both span the vector space of $8 \times 8$ matrices. In these bases,

$$
\begin{aligned}
& \stackrel{\circ}{\Gamma}_{m_{1} \ldots m_{7}}=-i{\stackrel{\circ}{m_{1} \ldots m_{7}}} \mathbb{I}_{8 \times 8}, \quad \quad \Gamma_{m_{1} \ldots m_{7}}=-i \epsilon_{m_{1} \ldots m_{7}} \mathbb{I}_{8 \times 8}, \\
& \stackrel{\circ}{\Gamma}_{m_{1} \ldots m_{6}}=-i{\stackrel{\circ}{m_{1} \ldots m_{7}}}^{\stackrel{\circ}{\Gamma}^{m_{7}},} \quad \quad \Gamma_{m_{1} \ldots m_{6}}=-i \epsilon_{m_{1} \ldots m_{7}} \Gamma^{m_{7}} \text {, } \\
& \stackrel{\circ}{\Gamma}_{m_{1} \ldots m_{5}}=\frac{i}{2} \stackrel{\circ}{\eta}_{m_{1} \ldots m_{7}} \stackrel{\circ}{\Gamma}^{m_{6} m_{7},} \quad \quad \Gamma_{m_{1} \ldots m_{5}}=\frac{i}{2} \epsilon_{m_{1} \ldots m_{7}} \Gamma^{m_{6} m_{7}} \text {, } \\
& \stackrel{\circ}{\Gamma}_{m_{1} \ldots m_{4}}=\frac{i}{3 !} \stackrel{\circ}{\eta}_{m_{1} \ldots m_{7}} \stackrel{\circ}{\Gamma}^{m_{5} \cdots m_{7}}, \quad \quad \Gamma_{m_{1} \ldots m_{4}}=\frac{i}{3 !} \epsilon_{m_{1} \ldots m_{7}} \Gamma^{m_{5} \cdots m_{7}} .
\end{aligned}
$$

Beside the Clifford algebra, the $\Gamma$-matrices satisfy the useful relations

$$
\begin{array}{lll}
\operatorname{Tr}\left(\stackrel{\circ}{\Gamma}^{m} \stackrel{\circ}{\Gamma}^{n}\right)=8 \stackrel{\circ}{g}^{m n}, & \operatorname{Tr}\left(\stackrel{\circ}{\Gamma}^{m} \stackrel{\circ}{\Gamma}^{n p}\right)=0, & \operatorname{Tr}\left(\stackrel{\circ}{\Gamma}^{m n} \Gamma_{p q}^{\circ}\right)=-16 \delta_{p q}^{m n}, \\
\operatorname{Tr}\left(\Gamma^{m} \Gamma^{n}\right)=8 g^{m n}, & \operatorname{Tr}\left(\Gamma^{m} \Gamma^{n p}\right)=0, & \operatorname{Tr}\left(\Gamma^{m n} \Gamma_{p q}\right)=-16 \delta_{p q}^{m n} .
\end{array}
$$

The Killing spinors define a set of Killing vectors and their derivatives,

$$
\begin{aligned}
K_{m}{ }^{I J} & =i \bar{\eta}^{I} \stackrel{\circ}{\Gamma}_{m} \eta^{J}, \\
K_{m n}{ }^{I J} & =\bar{\eta}^{I} \stackrel{\circ}{\Gamma}_{m n} \eta^{J}, \\
K_{m_{1} \cdots m_{5}}{ }^{I J} & =i \bar{\eta}^{I} \stackrel{\circ}{\Gamma}_{m_{1} \cdots m_{5}} \eta^{J} .
\end{aligned}
$$

Using eq. (A.3), one verifies that $K_{m n}{ }^{I J}$ is indeed, proportional to the derivative of $K_{m}{ }^{I J}$,

$$
\stackrel{\circ}{D}_{n} K_{m}{ }^{I J}=m_{7} K_{m n}{ }^{I J}, \quad \stackrel{\circ}{D}_{p} K_{m n}{ }^{I J}=2 m_{7} \stackrel{\circ}{g}_{p[m} K_{n]}{ }^{I J} .
$$

Using eq. (A.8), one also finds that $K_{m_{1} \cdots m_{5}}{ }^{I J}$ is the (seven dimensional) dual to $K_{m n}{ }^{I J}$,

$$
K_{m_{1} \cdots m_{5}}=-\frac{1}{2} \grave{\eta}_{m_{1} \cdots m_{7}} K^{m_{6} m_{7} I J} .
$$

Note that curved seven dimensional indices of the Killing vectors and their derivatives are always raised and lowered with the background metric $\stackrel{\circ}{g}_{m n}$.

The following bi-linears in the $\Gamma$-matrices represent a basis for (anti-)selfdual SU(8) tensors on the deformed seven-sphere:

$$
\begin{array}{rlll}
\text { selfdual : } & \Gamma_{m[A B} \Gamma_{C D]}^{m}, & \Gamma_{m n}[A B \\
\text { anti - selfdual : } & \Gamma_{C D]}^{[m n}, & \Gamma_{[A B}^{m} \Gamma_{C D}^{p]} . &
\end{array}
$$


On the background, there is the respective basis of (anti-)selfdual SL(8) tensors in terms of the Killing bi-linears, i.e.

$$
\begin{array}{rlll}
\text { selfdual : } & K_{m}{ }^{[I J} K^{m K L]}, & K_{m n}{ }^{[I J} K^{n K L]}, & K_{m}{ }^{[I J} K_{n}{ }^{K L]} \\
\text { anti - selfdual : } & K_{[m n}{ }^{[I J} K_{p]}{ }^{K L]} . &
\end{array}
$$

These bi-linears satisfy further useful relations [16, 32],

$$
\begin{aligned}
\Gamma_{m[A B} \Gamma_{C D]}^{m}= & \Gamma_{m A B} \Gamma^{m}{ }_{C D}+2 \delta_{C D}^{A B}, \\
\Gamma_{m n[A B} \Gamma_{C D]}^{n}= & \frac{1}{2}\left(\Gamma_{m n} A B \Gamma_{C D}^{n}+\Gamma_{m n C D} \Gamma^{n}{ }_{A B}\right), \\
\Gamma_{[A B}^{m n} \Gamma_{C D]}^{p}= & -\frac{1}{3} g^{p[m} \Gamma^{n] q}{ }_{[A B} \Gamma_{q C D]}+\Gamma^{[m n}{ }_{[A B} \Gamma_{C D]}^{p]}, \\
\Gamma_{[A B}^{m n} \Gamma_{C D]}^{p q}= & -2 g^{m[p} \Gamma^{q]}{ }_{[A B} \Gamma_{C D]}^{n}+2 g^{n[p} \Gamma^{q]}{ }_{[A B} \Gamma_{C D]}^{m}+ \\
& +\frac{2}{3} g^{m[p} g^{q] n} \Gamma_{r[A B} \Gamma_{C D]}^{r}+\Gamma_{[A B}^{[m n} \Gamma_{C D]}^{p q]}
\end{aligned}
$$

as well as

$$
\begin{aligned}
K_{m}{ }^{[I J} K^{m K L]}= & K_{m}{ }^{I J} K^{m K L}-2 \delta_{K L}^{I J}, \\
K_{m n}{ }^{[I J} K^{n K L]}= & \frac{1}{2}\left(K_{m n}{ }^{I J} K^{n K L}+K_{m n}{ }^{K L} K^{n I J}\right), \\
K_{m n}{ }^{[I J} K_{p}{ }^{K L]}= & -\frac{1}{3} \stackrel{\circ}{p}_{p[m} K_{n] q}{ }^{[I J} K^{q K L]}+K_{[m n}{ }^{[I J} K_{p]}{ }^{K L]}, \\
K_{m n}{ }^{[I J} K_{p q}{ }^{K L]}= & 2 \stackrel{\circ}{m}_{m[p} K_{q]}^{[I J} K_{n}{ }^{K L]}-2 \stackrel{\circ}{g}\left[p_{n\left[{ }^{[}{ }^{[I J} K_{m} K L\right]}\right. \\
& -\frac{2}{3} \stackrel{\circ}{g}_{m[p} \stackrel{\circ}{q}_{q] n} K_{r}^{[I J} K^{r K L]}+K_{[m n}^{[I J} K_{p q]}{ }^{K L]} .
\end{aligned}
$$

One has furthermore [32]

$$
\begin{aligned}
& \Gamma_{m n A B} \Gamma_{C D}^{n}-\Gamma_{m n C D} \Gamma_{A B}^{n}=-4\left(\delta_{C[A} \Gamma_{m B] D}-\delta_{D[A} \Gamma_{m B] C}\right), \\
& K_{m n}{ }^{I J} K^{n K L}-K_{m n}{ }^{n L} K^{n I J}=-8 \delta^{[I}{ }_{[K} K_{m}{ }^{J]}{ }_{L]} .
\end{aligned}
$$

The bases in eqs. (A.17)-(A.20) are in some sense 'orthogonal'. Indeed, one has

$$
\begin{aligned}
& \Gamma_{[A B}^{m} \Gamma_{C D}^{n} \Gamma^{p}{ }_{A B} \Gamma^{q}{ }_{C D}=16 g^{m(p} g^{n q)}, \\
& \Gamma_{m p[A B} \Gamma^{p}{ }_{C D]} \Gamma_{n q A B} \Gamma^{q} C D=-192 g_{m n}, \\
& \Gamma^{[m n}{ }_{[A B} \Gamma^{p]}{ }_{C D]} \Gamma_{[q r A B} \Gamma_{s] C D}=-32 \delta_{q r s}^{m n p}, \\
& K_{m}{ }^{[I J} K_{n}{ }^{K L]} K_{p}{ }^{I J} K_{q}{ }^{K L}=16 \stackrel{\circ}{g}_{m(p}\left(\stackrel{\circ}{g}_{n q}\right), \\
& K_{m p}{ }^{[I J} K^{p K L]} K_{n q}{ }^{I J} K^{q K L}=192 \stackrel{\circ}{g}_{m n}, \\
& K_{[m n}{ }^{[I J} K_{p]}{ }^{K L]} K^{[q r I J} K^{s] K L}=32 \delta_{m n p}^{q r s} .
\end{aligned}
$$

whereas all other contractions, such as

$$
\Gamma_{[A B}^{m} \Gamma_{C D]}^{n} \Gamma_{p q A B} \Gamma^{q} C D=0, \quad K_{m}^{[I J} K_{n}{ }^{K L]} K_{p q}{ }^{I J} K^{q K L}=0
$$


vanish identically.

Finally, it is convenient to define the selfdual tensor

$$
K^{I J K L}=K_{m}^{[I J} K^{m K L]},
$$

which satisfies $[26]$

$$
\begin{aligned}
& K^{I J K P} K_{L M N P}=6 \delta_{L M N}^{I J K}+9 \delta^{[I}{ }_{[L} K^{J K]}{ }_{M N]}, \\
& \left.K^{[I J K L} K^{M] N P Q}=\frac{1}{5} \epsilon^{I J K L M N P Q}+12 K^{[I J K}{ }_{[N} \delta^{L}{ }_{P} \delta^{M]} Q\right], \\
& \left.\left.K^{m I J} K^{n K L} K_{m n}{ }^{M N}=8 \delta_{[K}^{[I} \delta^{J][M} \delta^{N]} L\right]+4 \delta_{[I}^{[M} K^{N]} J\right] K L \\
& +4 \delta^{[K}{ }_{[M} K^{L]}{ }_{N] I J}-4 \delta^{[I}{ }_{[K} K^{J]}{ }_{L] M N} .
\end{aligned}
$$

Open Access. This article is distributed under the terms of the Creative Commons Attribution License (CC-BY 4.0), which permits any use, distribution and reproduction in any medium, provided the original author(s) and source are credited.

\section{References}

[1] T. Kaluza, On the Problem of Unity in Physics, Sitzungsber. Preuss. Akad. Wiss. Berlin (Math. Phys.) 1921 (1921) 966 [inSPIRE].

[2] O. Klein, Quantum Theory and Five-Dimensional Theory of Relativity. (In German and English), Z. Phys. 37 (1926) 895 [inSPIRE].

[3] E. Cremmer and B. Julia, The $N=8$ Supergravity Theory. 1. The Lagrangian, Phys. Lett. B 80 (1978) 48 [INSPIRE].

[4] B. de Wit and H. Nicolai, N=8 Supergravity, Nucl. Phys. B 208 (1982) 323 [inSPIRE].

[5] E. Cremmer, B. Julia and J. Scherk, Supergravity Theory in Eleven-Dimensions, Phys. Lett. B 76 (1978) 409 [INSPIRE].

[6] W. Nahm, Supersymmetries and their Representations, Nucl. Phys. B 135 (1978) 149 [INSPIRE].

[7] F. Englert, Spontaneous Compactification of Eleven-Dimensional Supergravity, Phys. Lett. B 119 (1982) 339 [INSPIRE].

[8] B. Biran, F. Englert, B. de Wit and H. Nicolai, Gauged $N=8$ Supergravity and Its Breaking From Spontaneous Compactification, Phys. Lett. B 124 (1983) 45 [Erratum ibid. B 128 (1983) 461] [INSPIRE].

[9] B. de Wit and H. Nicolai, The Consistency of the $S^{7}$ Truncation in D $=11$ Supergravity, Nucl. Phys. B 281 (1987) 211 [inSPIRE].

[10] M. Duff and C. Pope, Kaluza-Klein supergravity and the seven sphere, lectures given at the September School on Supergravity and Supersymmetry, Trieste, Italy, 6-18 September 1982 [INSPIRE].

[11] H. Nicolai and K. Pilch, Consistent Truncation of $D=11$ Supergravity on $A d S_{4} \times S^{7}, J H E P$ 03 (2012) 099 [arXiv: 1112.6131] [INSPIRE].

[12] B. de Wit and H. Nicolai, Deformations of gauged $\mathrm{SO}(8)$ supergravity and supergravity in eleven dimensions, JHEP 05 (2013) 077 [arXiv:1302.6219] [INSPIRE]. 
[13] H. Godazgar, M. Godazgar and H. Nicolai, Generalised geometry from the ground up, JHEP 02 (2014) 075 [arXiv: 1307.8295] [INSPIRE].

[14] H. Godazgar, M. Godazgar and H. Nicolai, Nonlinear Kaluza-Klein theory for dual fields, Phys. Rev. D 88 (2013) 125002 [arXiv:1309.0266] [INSPIRE].

[15] B. de Wit, H. Nicolai and N.P. Warner, The Embedding of Gauged $N=8$ Supergravity Into $d=11$ Supergravity, Nucl. Phys. B 255 (1985) 29 [INSPIRE].

[16] H. Godazgar, M. Godazgar and H. Nicolai, Testing the non-linear flux ansatz for maximal supergravity, Phys. Rev. D 87 (2013) 085038 [arXiv: 1303.1013] [InSPIRE].

[17] H. Godazgar, M. Godazgar, O. Krüger, H. Nicolai and K. Pilch, An $\mathrm{SO}(3) \times \mathrm{SO}(3)$ invariant solution of $D=11$ supergravity, JHEP 01 (2015) 056 [arXiv:1410.5090] [INSPIRE].

[18] K. Pilch, A. Tyukov and N.P. Warner, Flowing to Higher Dimensions: A New Strongly-Coupled Phase on M2 Branes, JHEP 11 (2015) 170 [arXiv:1506.01045] [INSPIRE].

[19] K. Pilch, A. Tyukov and N.P. Warner, $\mathcal{N}=2$ Supersymmetric Janus Solutions and Flows: From Gauged Supergravity to M-theory, JHEP 05 (2016) 005 [arXiv: 1510.08090] [INSPIRE].

[20] O. Varela, The complete $D=11$ embedding of $\mathrm{SO}(8)$ supergravity, arXiv:1512.04943 [INSPIRE].

[21] H. Nicolai, P.K. Townsend and P. van Nieuwenhuizen, Comments on eleven-dimensional supergravity, Lett. Nuovo Cim. 30 (1981) 315 [INSPIRE].

[22] P.G.O. Freund and M.A. Rubin, Dynamics of Dimensional Reduction, Phys. Lett. B 97 (1980) 233 [INSPIRE].

[23] B. de Wit and H. Nicolai, A New $\mathrm{SO}(7)$ Invariant Solution of $d=11$ Supergravity, Phys. Lett. B 148 (1984) 60 [INSPIRE].

[24] H. Godazgar, M. Godazgar and H. Nicolai, Einstein-Cartan Calculus for Exceptional Geometry, JHEP 06 (2014) 021 [arXiv:1401.5984] [INSPIRE].

[25] E. Witten, Search for a Realistic Kaluza-Klein Theory, Nucl. Phys. B 186 (1981) 412 [INSPIRE].

[26] H. Godazgar, M. Godazgar, O. Krüger and H. Nicolai, Consistent 4-form fluxes for maximal supergravity, JHEP 10 (2015) 169 [arXiv:1507.07684] [INSPIRE].

[27] C.M. Hull and N.P. Warner, Noncompact Gaugings From Higher Dimensions, Class. Quant. Grav. 5 (1988) 1517 [INSPIRE].

[28] W.H. Baron and G. Dall'Agata, Uplifting non-compact gauged supergravities, JHEP 02 (2015) 003 [arXiv: 1410.8823] [INSPIRE].

[29] K. Lee, C. Strickland-Constable and D. Waldram, Spheres, generalised parallelisability and consistent truncations, arXiv:1401.3360 [INSPIRE].

[30] F. Ciceri, B. de Wit and O. Varela, IIB supergravity and the $E_{6(6)}$ covariant vector-tensor hierarchy, JHEP 04 (2015) 094 [arXiv: 1412.8297] [INSPIRE].

[31] A. Baguet, O. Hohm and H. Samtleben, Consistent Type IIB Reductions to Maximal 5D Supergravity, Phys. Rev. D 92 (2015) 065004 [arXiv:1506.01385] [InSPIRE].

[32] B. de Wit and H. Nicolai, $d=11$ Supergravity With Local SU(8) Invariance, Nucl. Phys. B 274 (1986) 363 [INSPIRE]. 